\title{
Optimal investment with high-watermark performance fee
}

\author{
Karel Janeček * and Mihai Sîrbu ${ }^{\dagger}$ \\ January 5, 2011
}

\begin{abstract}
We consider the problem of optimal investment and consumption when the investment opportunity is represented by a hedge-fund charging proportional fees on profit. The value of the fund evolves as a geometric Brownian motion and the performance of the investment and consumption strategy is measured using discounted power utility from consumption on infinite horizon. The resulting stochastic control problem is solved using dynamic programming arguments. We show by analytical methods that the associated Hamilton-Jacobi-Bellman equation has a smooth solution, and then obtain the existence and representation of the optimal control in feedback form using verification arguments.
\end{abstract}

Keywords: optimal investment, stochastic control, high-watermark, viscosity solutions, verification lemma

Mathematics Subject Classification (2010): 91G10, 93E20, 49L20 JEL classification: G110

\section{Introduction}

Investment funds (hedge funds, for example) charge different kinds of fees to the investors. Some of the most typical fees are fixed proportions of the total size of investment, paid as a percentage per year, and also, very often, a provision to charge a fixed proportion of the profit on investment, whenever profit is made. The industry standard for hedge-funds is the so called " $2 / 20$-rule": $2 \%$ per year of the total investment and $20 \%$ percent of the additional profits are paid to the fund manager. Other common fee structures are $1 \%$ per year and up to $50 \%$ of the profits. In order to pay profit-fees, the total size of the achieved profit of the agent is followed. Whenever the maximum to day, the so called high-water mark, exceeds the previously attained historic maximum, the fixed proportion of the profit (relative to the previous maximum) is charged by the fund manager. The goal of the present paper is to model optimal investment and consumption in such a fund as a stochastic control problem and analyze it using dynamic programming.

While optimal investment in one or more assets, usually having the meaning of stocks, with the possibility of buying/selling them for the same price and also the possibility of borrowing/lending cash for the same rate is a classical topic and well understood in a very general mathematical framework, there is also a large more recent literature related to the investment in markets with frictions. Transaction costs is the prime example of market imperfections: the papers [5], [16], [17] and [10] are just a few examples of the growing literature on the topic. We study here a new kind of possible frictions affecting investment, namely the fees that are paid on the profit of the investment in a hedge-fund as

${ }^{*}$ RSJ algorithmic trading and Charles University, Prague. E-mail address: Karel.Janecek@rsj.cz. This author would like to thank Professor Karl Kunish, University of Graz, Austria, for his help and invaluable lecture dealing with the numerical algorithms for solving the partial differential equations in this paper

${ }^{\dagger}$ University of Texas at Austin, Department of Mathematics, 1 University Station C1200, Austin, TX, 78712. E-mail address: sirbu@math.utexas.edu. The research of this author was supported in part by the National Science Foundation under Grant DMS 0908441. 
described above, the so called high -watermark profit fees. Mathematically, this problem does not fit in the existing literature related to investment with frictions/constraints, because the existing literature mainly addresses the constraints associated to investing directly in the equities.

A number of papers recently considered the presence of high-watermark fees from the point of view of the fund manager. Guasoni and Obloj [9] study the problem of a fund manager with large time horizon and constant investment opportunity, who maximizes his/her expected utility from accumulated profit fees. This paper is closest to our work since the equation describing the evolution of the fund share in [9] has similar path-wise solutions to the state equation describing the evolution of the investor's wealth in the present work. However, the stochastic control problem in [9] is different from ours. Panagea and Westerfield [14] consider a risk-neutral fund manager maximizing the present value of future fees. Grossman, Ingersol Jr. and Ross [7] study the incentive that the high-watermark fees represent for the fund manager, and provide a closed form solution for the high-watermark provision as a claim on the investor's wealth. The effect of the high-watermark fees on the investment manager's behavior is to seek long term growth, which is consistent with the investor's objective, see [1], [2].

To the best of our knowledge, the effect of the high-watermark fees on the investor decisions is not yet addressed in the literature. In order to understand this effect, we model the fund share/unit price exogenously. The investor is making the trading decisions. The fund charges the profit fee for the service of offering a positive expected return for the investor. This modeling approach describes well the real-world applications. We are interested in analyzing the behavior of the investor who can only invest in the fund and the money market. The main goal is to understand how the high-watermark fee changes the behavior of the investor, compared to the case when the fund has an identical evolution but no profit fees are paid.

The main findings of the paper can be summarized as follows: first, the optimal investment problem can be modeled as the stochastic control of a two-dimensional reflected diffusion. Then, the control problem is well posed (there exist unique optimal controls), and solving the problem can be reduced to finding the smooth solution of the associated Hamilton-Jacobi-Bellman equation. The numerical results show that, compared to the case of no fee (the classical Merton problem), the fees change the behavior of the investor with reasonable levels of risk aversion by making the investor invest more in the fund when the high-watermark is reached, in order to increase the high-watermark and avoid paying future fees (see Remark 4.3). When the investor is far from paying fees, the investment/consumption strategies are very close to those corresponding to paying no fees. This is proved analytically and can also be seen from the numerics.

Since our model consists in controlling a reflected diffusion, where the reflection is actually coming from a running maximum, our problem is technically related to the problem of optimal investment with draw-down constrains in [8], [4], [15] and [6]. However, unlike the papers [15] and [6], our problem does not have a closed form solution. Therefore we need to prove that the Hamilton-Jacobi-Bellman equation has a smooth solution. We prove this analytically, using Perron's method to obtain existence of a viscosity solution and then upgrade regularity. Our analysis allows us to avoid proving the Dynamic Programming Principle, and the measurability technicalities associated with it. The reason why there is no closed form solution, as opposed to the problem of draw-down constraints in [15] and [6] is the fact that consumption is present in the running-maximum. This is an important part of our model, and comes form the fact that fees are assessed based on profit, independently of the consumption choices of the investor.

The paper is organized as follows: in Section 2.1 we model optimal investment/consumption as a stochastic control problem. In Section 3 we present the main results. We analyze the impact of the high-watermark fee in Section 4 and present the conclusions. Since the control problem does not have a closed-form solution, we complement the analysis with some numerical case-studies in Subsection 4.3. For convenience of the reader, we defer the technical proofs to Section 5. More precisely, we first show in Subsection 5.1 that the Hamilton-Jacobi-Bellman equation has a viscosity solution by Perron's method, and then we upgrade its regularity in Subsection 5.2. We go over the verification arguments in Subsection 5.3. 


\section{The model}

\subsection{A general model of profits from dynamic investment in a hedge-fund}

Consider an investor who chooses as investment vehicle a risky fund (hedge-fund) with share/unit price $F_{t}$ at time $t$. We assume that the investor can freely move money in and out of the risky fund and therefore continuously rebalance her investment. If the investor chooses to hold $\theta_{t}$ capital in the fund at time $t$ and no fees of any kind are imposed, then her accumulated profit from investing in the fund evolves as

$$
\left\{\begin{array}{l}
d P_{t}=\theta_{t} \frac{d F_{t}}{F_{t}}, 0 \leq t<\infty \\
P_{0}=0
\end{array}\right.
$$

Assume now that a proportion $\lambda>0$ of the profits achieved by investing in the fund is paid by the investor to the fund manager. The fee is a commission to the fund manager for offering an investment opportunity for the investor (usually with a positive expected return). The fund manager keeps track of the accumulated profit that the investor made by holding the fund shares. More precisely, the manager tracks the high-watermark of the investor's achieved profit

$$
M_{t} \triangleq \sup _{0 \leq s \leq t} P_{s}
$$

Anytime the high-water mark increases, a $\lambda$ percentage of this increase is paid to the fund manager, i.e., $\lambda \Delta M_{t}=\lambda\left(M_{t+\Delta t}-M_{t}\right)$ is paid by the investor to the manager in the interval $[t, t+\Delta t]$. Therefore, the evolution of the profit $P_{t}$ of the investor is given by

$$
\left\{\begin{array}{l}
d P_{t}=\theta_{t} \frac{d F_{t}}{F_{t}}-\lambda d M_{t}, \quad P_{0}=0 \\
M_{t}=\sup _{0 \leq s \leq t} P_{s}
\end{array}\right.
$$

We emphasize that the fund price process $F$ is exogenous to the investor.

Remark 2.1 1. There exist several kinds of hedge-funds. For some, the accounting for the purpose of assessing fees is done per share, so for each share bought/sold fees are computed separately, and the accounting is based on the high-watermark of the fund as a whole. Alternatively, the accounting is done per personal account of the investor, who is actually allowed to rebalance his portfolio, and fees are assessed the way described above.

2. While dynamic investment in a hedge-fund is the original motivation for our equation (2), this is also a good model of taxation. ${ }^{1}$ More precisely, this is a model of capital gains taxation at the rate $\lambda$, in case the taxes are paid at the time the gain is realized. Past losses are deducted from gains in order to asses taxes exactly the way described in equation (2). If we decide to consider this interpretation of the model, then $F$ has the meaning of any risky/asset (stock) and multiple assets $F$ can be considered.

While this is usually not done in practice, for mathematical reasons related to dynamic programming, we can assume that the investor is given an initial high-watermark for her profits, $i \geq 0$. This way, the profits of the investor will not be taxed before $P$ reaches at least value $i$. More precisely, for fixed $i \geq 0$, we have the evolution of the profits $P$ (and the high-watermark of the profits $M$ ) given by

$$
\left\{\begin{array}{l}
d P_{t}=\theta_{t} \frac{d F_{t}}{F_{t}}-\lambda d M_{t}, \quad P_{0}=0 \\
M_{t}=\sup _{0 \leq s \leq t}\left(P_{s} \vee i\right) .
\end{array}\right.
$$

Equation (2) is implicit, so the existence and uniqueness of the solution should be analyzed carefully. This is done in Proposition 2.1 below, and fortunately, can be done in closed form path-wise. A similar representation appears in the Appendix of Guasoni and Obloj [9]. The paper [9] studies a different

\footnotetext{
${ }^{1}$ We thank Paolo Guasoni who pointed out this interpretation.
} 
optimization problem, related to maximizing utility of the fund manager as opposed to the utility of the investor in our case. However, their state equation is similar to our equation (2), so we resort to the same path-wise representation.

Proposition 2.1 Assume that the share/unit prices process $F$ is a continuous and strictly positive semimartingale. Assume also that the predictable processes $\theta$ is such that the accumulated profit process corresponding to the trading strategy $\theta$, in case no profit fees are imposed, namely

$$
I_{t}=\int_{0}^{t} \theta_{u} \frac{d F_{u}}{F_{u}}, \quad 0 \leq t<\infty
$$

is well defined. Then equation (2) has a unique solution, which can be represented path-wise by

$$
\begin{aligned}
& P_{t}=I_{t}-\frac{\lambda}{1+\lambda} \sup _{0 \leq s \leq t}\left[I_{s}-i\right]^{+}, \quad 0 \leq t<\infty, \\
& M_{t}=i+\frac{1}{1+\lambda} \max _{0 \leq s \leq t}\left[I_{s}-i\right]^{+}, \quad 0 \leq t<\infty .
\end{aligned}
$$

Proof: Equation (2) can be rewritten as

$$
\left(P_{t}-i\right)+\lambda \sup _{0 \leq s \leq t}\left[P_{s}-i\right]^{+}=I_{t}-i, \quad 0 \leq t<\infty .
$$

Taking the positive part and the supremum on both sides we get

$$
(1+\lambda)\left(M_{t}-i\right)=(1+\lambda) \sup _{0 \leq s \leq t}\left[P_{s}-i\right]^{+}=\sup _{0 \leq s \leq t}\left[I_{s}-i\right]^{+}, \quad 0 \leq t<\infty .
$$

Replacing this equality in (2) we finish the proof of uniqueness. Existence follows easily, by checking that the process in $(3)$ is a solution of $(2) . \diamond$

\subsection{Optimal investment and consumption in a special model}

We now assume that the investor starts with initial capital $x>0$ and the only additional investment opportunity is the money market paying zero interest rate. The investor is given the initial highwatermark $i \geq 0$ for her profits. We further assume that the investor consumes at a rate $\gamma_{t}>0$ per unit of time. Consumption can be made either from the money market account or from the risky fund, as wealth can be moved from one another at any time. We denote by

$$
C_{t} \triangleq \int_{0}^{t} \gamma_{s} d s, 0 \leq t<\infty
$$

the accumulated consumption. Since the money market pays zero interest rate, the wealth $X_{t}$ of the investor at time $t$ is given as initial wealth plus profit from the fund minus accumulated consumption

$$
X_{t}=x+P_{t}-C_{t}, 0 \leq t<\infty .
$$

Taking this into account, the high-watermark of investor's achieved profit can be computed by tracking her wealth and accumulated consumption. More precisely, the high-watermark can be represented as

$$
M_{t}=\sup _{0 \leq s \leq t}\left[\left(X_{s}+C_{s}-x\right) \vee i\right]+=i+\sup _{0 \leq s \leq t}\left[\left\{X_{s}+C_{s}\right\}-n\right]^{+},
$$

where $n \triangleq x+i \geq x>0$. The investor's wealth follows the evolution equation

$$
\left\{\begin{array}{l}
d X_{t}=\theta_{t} \frac{d F_{t}}{F_{t}}-\gamma_{t} d t-\lambda d M_{t}, \quad X_{0}=x \\
M_{t}=i+\sup _{0 \leq s \leq t}\left[\left\{X_{s}+\int_{0}^{s} \gamma_{u} d u\right\}-n\right]^{+} .
\end{array}\right.
$$


So far, this is a general model of investment/consumption in a hedge-fund, which is also a good model of taxation as pointed out in Remark 2.1.

In what follows, we choose a particular model for which we solve the problem of optimal investment and consumption by dynamic programming. More precisely, we assume that the fund share/unit price $F$ evolves as a geometric Brownian motion, which means

$$
\frac{d F_{t}}{F_{t}}=\alpha d t+\sigma d W_{t}, \quad 0 \leq t<\infty
$$

Here $\left(W_{t}\right)_{0 \leq t<\infty}$ is a Brownian motion on the filtered probability space $\left(\Omega, \mathcal{F},\left(\mathcal{F}_{t}\right)_{0 \leq t<\infty}, \mathbb{P}\right)$, where the filtration $\left(\mathcal{F}_{t}\right)_{0 \leq t<\infty}$ satisfies the usual conditions. With these notation, equation $(5)$ for the wealth of the investor becomes

$$
\left\{\begin{array}{l}
d X_{t}=\left(\theta_{t} \alpha-\gamma_{t}\right) d t+\theta_{t} \sigma d W_{t}-\lambda d M_{t}, \quad X_{0}=x \\
M_{t}=i+\sup _{0 \leq s \leq t}\left[\left\{X_{s}+\int_{0}^{s} \gamma_{u} d u\right\}-n\right]^{+} .
\end{array}\right.
$$

In order to use dynamic programming, we want to represent the control problem using a state process of minimal dimension. As usual, the wealth $X$ has to be a part of the state. Simply using $(X, M)$ as state is not a possibility, since $M$ does not contain the information on past consumption, just of past profits. In order to choose the additional state(s), we observe that the fee is being paid as soon as the current profit $P_{t}=X_{t}+C_{t}-x$ (current wealth plus accumulated consumption minus initial wealth) hits the high-watermark $M_{t}=i+\sup _{0 \leq s \leq t}\left[\left(X_{t}+C_{t}\right)-n\right]^{+}$. In other words, fees are paid whenever

$$
X_{t}+C_{t}-n=\sup _{0 \leq s \leq t}\left[\left(X_{t}+C_{t}\right)-n\right]^{+},
$$

which is the same as $X=N$ for

$$
N_{t} \triangleq n+\sup _{0 \leq s \leq t}\left[\left(X_{s}+C_{s}\right)-n\right]^{+}-C_{t}=\sup _{0 \leq s \leq t}\left(\left\{X_{s}+C_{s}\right\} \vee n\right)-C_{t} \geq X_{t} .
$$

We now choose as state process the two-dimensional process $(X, N)$ which satisfies $X \leq N$ and is reflected whenever $X=N$. The controlled state process $(X, N)$ follows the evolution

$$
\left\{\begin{array}{l}
d X_{t}=\left(\theta_{t} \alpha-\gamma_{t}\right) d t+\theta_{t} \sigma d W_{t}-\lambda\left(d N_{t}+\gamma_{t} d t\right), \quad X_{0}=x \\
N_{t}=\sup _{0 \leq s \leq t}\left(\left\{X_{s}+\int_{0}^{s} \gamma_{u} d u\right\} \vee n\right)-\int_{0}^{t} \gamma_{u} d u
\end{array}\right.
$$

Equation (7) is again implicit, as is equation (2). The path-wise representation in Proposition 2.1 can be easily translated into a path-wise solution for (7). More precisely, we have the following proposition, whose proof is a direct consequence of Proposition 2.1, so we omit it.

Proposition 2.2 Assume that the predictable processes $(\theta, \gamma)$ satisfy the following integrability property:

$$
\mathbb{P}\left(\int_{0}^{t}\left(\left|\theta_{u}\right|^{2}+\gamma_{u}\right) d u<\infty, \quad(\forall) \quad 0 \leq t<\infty\right)=1
$$

Denote by

$$
Y_{t}=\int_{0}^{t} \theta_{u}\left(\alpha d u+\sigma d W_{u}\right), \quad C_{t}=\int_{0}^{t} \gamma_{u} d u, \quad 0 \leq t<\infty,
$$

the accumulated profit process corresponding to the trading strategy $\theta$, in case no profit fees are imposed and the accumulated consumption. Equation (7) has a unique solution, which can be represented pathwise by

$$
\begin{gathered}
X_{t}=x+Y_{t}-C_{t}-\frac{\lambda}{1+\lambda} \sup _{0 \leq s \leq t}\left[Y_{s}-i\right]^{+}, \quad 0 \leq t<\infty, \\
N_{t}=n+\frac{1}{1+\lambda} \sup _{0 \leq s \leq t}\left[Y_{s}-i\right]^{+}-C_{t}, \quad 0 \leq t<\infty .
\end{gathered}
$$


The high-watermark is computed as

$$
M_{t}=i+\frac{1}{1+\lambda} \max _{0 \leq s \leq t}\left[Y_{s}-i\right]^{+} .
$$

Fix an initial capital $x>0$ and an initial high-watermark of profits $i \geq 0$. Recall that $n \triangleq x+i \geq x$. An investment/consumption strategy $(\theta, \gamma)$ is called admissible with respect to the initial data $(x, n)$ if it satisfies the integrability conditions (8), the consumption stream is positive $\left(\gamma_{t} \geq 0\right)$ and the wealth is strictly positive: $X_{t}>0$ for all times $t$. We denote by $\mathcal{A}(x, n)$ the set of all admissible strategies at $(x, n)$. Admissible strategies can be equivalently represented in terms of the proportions $\pi=\theta / X$ and $c=\gamma / X$. Surprisingly, we do not have a similar path-wise representation for $(X, N)$ in terms of $(\pi, c)$ as in Proposition 2.2, unless $c=0$ as in [9], when one can solve path-wise for $\log X$.

We model the preferences of the investor by the well known concept of expected utility from consumption. Namely, we consider a concave utility function $U:(0, \infty) \rightarrow \mathbb{R}$ to define the expected utility from consumption $\mathbb{E}\left[\int_{0}^{\infty} e^{-\beta t} U\left(c_{t}\right) d t\right]$. The discount factor $\beta>0$ accounts for either impatience or for the the idea that "whatever happens later matters less". In this model, the problem of optimal investment/consumption amounts to finding, for each fixed $(x, n)$, (the) optimal $(\theta, \gamma)$ in the optimization problem

$$
V(x, n) \triangleq \sup _{(\theta, \gamma) \in \mathcal{A}(x, n)} \mathbb{E}\left[\int_{0}^{\infty} e^{-\beta t} U\left(\gamma_{t}\right) d t\right], \quad 0<x \leq n .
$$

The function $V$ defined above is called the value function. We further assume that the agent has homogeneous preferences, meaning that the utility function $U$ has the particular form

$$
U(\gamma)=\frac{\gamma^{1-p}}{1-p}, \quad \gamma>0
$$

for some $p>0, p \neq 1$ called relative risk aversion coefficient.

Remark 2.2 In our framework, we can easily analyze the case when, in addition to the proportional profit-fee $\lambda$, the investor pays a continuous proportional fee with size $\nu>0$ (percentage of the wealth under investment management per units of time). In order to do this we just need to reduce the size of the excess return $\alpha$ by the proportional fee to $\alpha-\nu$ in the evolution of the fund share/unit.

Before we proceed, we would like to point out that, mathematically, the optimization problem (11) amounts to controlling a two-dimensional reflecting diffusion. More precisely, using the controls $(\theta, \gamma)$ the investor controls the diffusion $(X, N)$ in (7) which is restricted to the domain $\{0<x \leq n\}$ and is reflected on the diagonal $\{x=n\}$ in the direction given by the vector

$$
\tilde{r} \triangleq\left(\begin{array}{r}
-\lambda \\
1
\end{array}\right) \text {. }
$$

The reflection comes at the rate $d M_{t}$, where $M$ is the high-watermark. More precisely, equation (7) can be rewritten as

$$
d\left(\begin{array}{c}
X_{t} \\
N_{t}
\end{array}\right)=\left(\begin{array}{c}
\left(\theta_{t} \alpha-\gamma_{t}\right) d t+\theta_{t} \sigma d W_{t} \\
-\gamma_{t} d t
\end{array}\right)+\tilde{r} d M_{t}
$$

where

$$
\int_{0}^{t} \mathbb{I}_{\left\{X_{s} \neq N_{s}\right\}} d M_{s}=0
$$

Fortunately, the reflected equation (12) can be solved closed-form in terms of $(\theta, \gamma)$ by Proposition 2.2 .

The state equation (7) and therefore (12) are obtained based on the assumption that the money market pays zero interest, and hinges on the observation that tracking the wealth $X$ and the accumulated consumption $C$ allows to recover the high-watermark $M$. Using a different state variable, we can analyze more general models, including interest rates, hurdles, and additional investment opportunities. This is the subject of work in progress and will be presented in a forthcoming paper. The main goal of the present paper is to analyze the impact of fees on the investment/consumption strategies. 


\section{Dynamic Programming and Main Results}

\subsection{Formal Derivation of the HJB Equation}

The result below is a simple application of Itô's lemma and is easiest to see in the formulation (12) of the state equation (7).

Lemma 3.1 Let $v$ be a $C^{2}$ function on $\left\{(x, n) \in \mathbb{R}^{2} ; 0<x \leq n\right\}$ and $(X, N)$ a solution to the state equation (7) for $(\theta, \gamma) \in \mathcal{A}(x, n)$. Then

$$
\begin{aligned}
& d\left(\int_{0}^{t} e^{-\beta s} U\left(\gamma_{s}\right) d s+e^{-\beta t} v\left(X_{t}, N_{t}\right)\right)= \\
& e^{-\beta t}\left\{-\beta v\left(X_{t}, N_{t}\right)+U\left(\gamma_{t}\right)+\left(\alpha \theta_{t}-\gamma_{t}\right) v_{x}\left(X_{t}, N_{t}\right)+\frac{1}{2} \sigma^{2} \theta_{t}^{2} v_{x x}\left(X_{t}, N_{t}\right)-\gamma_{t} v_{m}\left(X_{t}, N_{t}\right)\right\} d t \\
& +e^{-\beta t}\left\{-\lambda v_{x}\left(X_{t}, N_{t}\right)+v_{n}\left(X_{t}, N_{t}\right)\right\} d M_{t}+\sigma e^{-\beta t} \theta_{t} v_{x}\left(X_{t}, N_{t}\right) d W_{t},
\end{aligned}
$$

where $M_{t}=N_{t}+\int_{0}^{t} \gamma_{s} d s-x$.

Taking into account that $d M$ is a singular measure with support on the set of times $\left\{t \geq 0: X_{t}=N_{t}\right\}$, we can formally write down the Hamilton-Jacobi-Bellman equation ((HJB) in the sequel):

$$
\begin{aligned}
\sup _{\gamma \geq 0, \theta}\left\{-\beta v+U(\gamma)+(\alpha \theta-\gamma) v_{x}+\frac{1}{2} \sigma^{2} \theta^{2} v_{x x}-\gamma v_{n}\right\} & =0, & \text { for } x>0, n>x, \\
-\lambda v_{x}(x, x)+v_{n}(x, x) & =0, & \text { for } x>0 .
\end{aligned}
$$

If we can find a smooth solution for the HJB then the optimal consumption will actually be given in feedback form by

$$
\hat{\gamma}(x, n)=I\left(v_{x}(x, n)+v_{n}(x, n)\right),
$$

where $I \triangleq U^{\prime-1}$ is the inverse of marginal utility. In addition, we expect the optimal amount invested in the fund $\hat{\pi}$ to be given by

$$
\hat{\theta}(x, n)=-\frac{\alpha}{\sigma^{2}} \frac{v_{x}(x, n)}{v_{x x}(x, n)}
$$

and that the smooth solution of the HJB is actually the value function, namely that

$$
v(x, n)=V(x, n), \quad 0<x \leq n,
$$

where $V$ was defined in (11).

\subsection{Dimension Reduction}

As mentioned above, we expect that the solution of the (HJB) is actually the value function for the optimization problem (11). Therefore, we can use the homotheticity property for the power utility function to reduce the number of variables to only one. More precisely, we expect that

$$
v(x, n)=x^{1-p} v\left(1, \frac{n}{x}\right) \triangleq x^{1-p} u(z) \text { for } z \triangleq \frac{n}{x} .
$$

In addition, instead of looking for the optimal amounts $\hat{\theta}(x, n)$ and $\hat{\gamma}(x, n)$ in (14) and (13) we look for the proportions

$$
\hat{c}(x, n)=\frac{I\left(v_{x}(x, n)+v_{n}(x, n)\right)}{x}
$$

and

$$
\hat{\pi}(x, n)=-\frac{\alpha}{\sigma^{2}} \frac{x v_{x}(x, n)}{x^{2} v_{x x}(x, n)}
$$


Since

$$
\begin{aligned}
v_{n}(x, n) & =u^{\prime}(z) \cdot x^{-p}, \\
v_{x}(x, n) & =\left((1-p) u(z)-z u^{\prime}(z)\right) \cdot x^{-p} \\
v_{x x}(x, n) & =\left(-p(1-p) u(z)+2 p z u^{\prime}(z)+z^{2} u^{\prime \prime}(z)\right) \cdot x^{-1-p},
\end{aligned}
$$

we get the one-dimensional HJB equation for $u(z), z>1$ :

$$
\begin{array}{r}
\sup _{c \geq 0, \pi}\left\{-\beta u+\frac{c^{1-p}}{1-p}+(\theta \alpha-c)\left((1-p) u-z u^{\prime}\right)-c u^{\prime}+\frac{1}{2} \pi^{2} \sigma^{2}\left(-p(1-p) u+2 p z u^{\prime}+z^{2} u^{\prime \prime}\right)\right\} \\
=0 \\
-\lambda(1-p) u(1)+(1+\lambda) u^{\prime}(1)=0 .
\end{array}
$$

We also expect that

$$
\lim _{z \rightarrow \infty} u(z)=\frac{1}{1-p} c_{0}^{-p}
$$

with $c_{0}$ given by (21) below, see (23).

The optimal investment proportion in (16) could therefore be expressed (provided we can find a smooth solution for the reduced HJB (18)) as

$$
\hat{\pi}(z)=\frac{\alpha}{p \sigma^{2}} \cdot \frac{(1-p) u-z u^{\prime}}{(1-p) u-2 z u^{\prime}-\frac{1}{p} z^{2} u^{\prime \prime}},
$$

and the optimal consumption proportion $\hat{c}$ in (15) would be given by

$$
\hat{c}(z)=\frac{\left(v_{x}+v_{n}\right)^{-\frac{1}{p}}}{x}=\left((1-p) u-(z-1) u^{\prime}\right)^{-\frac{1}{p}} .
$$

Remark 3.1 [The case when paying no fee, $\lambda=0$.$] This is the classical problem in Merton [12]$ and [13] and can be solved in closed form. The optimal investment and consumption proportions are constant. One can either take the solution from [12] and [13] or solve our equation (18) and then use (19) and (20) to obtain the same results.

More precisely, for $\lambda=0$, the optimal investment proportion is

$$
\pi_{0} \triangleq \frac{\alpha}{p \sigma^{2}}
$$

while the optimal consumption is given by

$$
c_{0} \triangleq \frac{\beta}{p}-\frac{1}{2} \frac{1-p}{p^{2}} \cdot \frac{\alpha^{2}}{\sigma^{2}} .
$$

The Merton value function (and solution of the HJB) equals

$$
v_{0}(x, n)=\frac{1}{1-p} c_{0}^{-p} x^{1-p}, \quad 0<x \leq n .
$$

It follows that, for $\lambda=0$,

$$
u_{0}(z)=\frac{1}{1-p} c_{0}^{-p}, \quad z \geq 1 .
$$

As can be easily seen from above, for the case $0<p<1$ an additional constraint needs to be imposed on the parameters in order to obtain a finite value function. This is equivalent to $c_{0}$ in (21) being strictly positive, which translates to the standing assumption

$$
\beta>\frac{1}{2} \frac{1-p}{p} \cdot \frac{\alpha^{2}}{\sigma^{2}}, \quad \text { if } \quad 0<p<1
$$




\subsection{Main Results}

We denote by

$$
\left(\mathcal{L}_{c, \theta} u\right)(z) \triangleq-\beta u+\frac{c^{1-p}}{1-p}+(\theta \alpha-c)\left((1-p) u-z u^{\prime}\right)-c u^{\prime}+\frac{1}{2} \theta^{2} \sigma^{2}\left(-p(1-p) u+2 p z u^{\prime}+z^{2} u^{\prime \prime}\right) .
$$

The (HJB) for $u$ can therefore be formally rewritten (with the implicit assumption that $-p(1-p) u+$ $2 p z u^{\prime}+z^{2} u^{\prime \prime}<0$ and $\left.(1-p) u-(z-1) u^{\prime}>0\right)$ as

$$
\left\{\begin{array}{l}
\left.\sup _{c \geq 0, \theta} \mathcal{L}_{c, \theta} u=-\beta u+\tilde{V}\left((1-p) u-(z-1) u^{\prime}\right)\right)-\frac{1}{2} \frac{\alpha^{2}}{\sigma^{2}} \frac{\left((1-p) u-z u^{\prime}\right)^{2}}{-p(1-p) u+2 p z u^{\prime}+z^{2} u^{\prime \prime}}=0, z>1 \\
-\lambda(1-p) u(1)+(1+\lambda) u^{\prime}(1)=0, \quad \lim _{z \rightarrow \infty} u(z)=\frac{1}{1-p} c_{0}^{-p}
\end{array}\right.
$$

where $\tilde{V}(y)=\frac{p}{1-p} y^{\frac{p-1}{p}}, \quad y>0$.

Let $w_{*}$ be the Merton value function for $\alpha=0$, i.e., with zero investment and optimized consumption. Then from $(21)$ and $(23)$ we get $c_{0}=\beta / p$, and

$$
w_{*}=\frac{1}{1-p}\left(\frac{\beta}{p}\right)^{-p}<u_{0} .
$$

Note that $w_{*}$ is also the unique non-trivial solution to the equation

$$
-\beta w+\tilde{V}((1-p) w))=0,
$$

and

$$
-\beta w+\tilde{V}((1-p) w))<0, \quad w_{*}<w \leq u_{0} .
$$

Next Theorem shows that the reduced (HJB) (26) has a classical solution which satisfies some additional properties.

Theorem 3.1 There exists a strictly increasing function $u$ which is $C^{2}$ on $[1, \infty)$, satisfies the condition $u(1)>w_{*}$ and

$$
-p(1-p) u+2 p z u^{\prime}+z^{2} u^{\prime \prime}<0, \quad(1-p) u-(z-1) u^{\prime}>0, \quad(1-p) u-z u^{\prime}>0, \quad z \geq 1,
$$

together with

$$
z u^{\prime}(z), z^{2} u^{\prime \prime}(z) \rightarrow 0, \text { as } z \rightarrow \infty
$$

and is a solution to (26).

The Proposition below shows that the so called closed-loop equation has a unique global solution.

Proposition 3.1 Fix $0<x \leq n$. Consider the feed-back proportions $\hat{\pi}(z)$ and $\hat{c}(z)$ defined in (19) and (20), where $u$ is the solution in Theorem 3.1. Define the feed-back controls

$$
\hat{\theta}(x, n) \triangleq x \hat{\pi}(n / x), \quad \hat{\gamma}(x, n) \triangleq x \hat{c}(n / x), \text { for } 0<x \leq n .
$$

The closed loop equation

$$
\left\{\begin{array}{l}
d X_{t}=\hat{\theta}\left(X_{t}, N_{t}\right)\left(\alpha d t+\sigma d W_{t}\right)-\hat{\gamma}\left(X_{t}, N_{t}\right) d t-\lambda\left(d N_{t}+\hat{\gamma}\left(X_{t}, N_{t}\right) d t\right), X_{0}=x \\
N_{t}=\sup _{0 \leq s \leq t}\left(\left\{X_{s}+\int_{0}^{s} \hat{\gamma}\left(X_{u}, N_{u}\right) d u\right\} \vee n\right)-\int_{0}^{t} \hat{\gamma}\left(X_{u}, N_{u}\right) d u,
\end{array}\right.
$$

has a unique strong global solution $(\hat{X}, \hat{N})$ such that $0<\hat{X} \leq \hat{N}$.

Next Theorem is the main result of the paper:

Theorem 3.2 Consider the solution $u$ in Theorem 3.1. For each $0<x \leq n$, the feedback proportions $(\hat{\pi}, \hat{c})$ in (19) and $(20)$ are optimal and

$$
u\left(\frac{n}{x}\right) x^{1-p} \triangleq v(x, n)=V(x, n) \triangleq \sup _{(\theta, \gamma) \in \mathcal{A}(x, n)} \mathbb{E}\left[\int_{0}^{\infty} e^{-\beta t} U\left(\gamma_{t}\right) d t\right] .
$$




\section{The Impact of the High-Watermark Fee on Investors}

In this section we analyze the impact of paying the high-watemark fee on the optimal investment and consumption strategy. In Subsection 4.1 we present some important qualitative properties of the optimal controls. In Subsection 4.2 we compare the optimal investment problem in the hedgefund with a high-watermark performance fee with the classical Merton problem. From the certainty equivalence point of view, paying the high-watermark fee is equivalent to either reducing the initial wealth (for the same excess return) or to reducing the excess return (for the same initial wealth), all other parameters being equal. Since the problem does not have a closed form solution, we present some numerical Examples in Subsection 4.3

\subsection{Some Qualitative Properties and Remarks}

The Proposition below analyzes the proportions $\hat{\pi}$ and $\hat{c}$, defined in (19) and (20), based on the solution $u$ of the (HJB) given by Theorem 3.1. Proposition 4.1 is needed in order to prove the existence and uniqueness of the solution of the closed-loop equation in Proposition 3.1. Once Proposition 3.1 and Theorem 3.2 are proved we actually know that $\hat{\pi}$ and $\hat{c}$ are the optimal proportions.

Proposition 4.1 The feedback controls $\hat{\pi}$ and $\hat{c}$ satisfy

$$
0<\hat{c}(z) \rightarrow c_{0}, \quad 0<\hat{\pi}(z) \rightarrow \pi_{0}, \quad z \rightarrow \infty
$$

and

$$
z \hat{c}^{\prime}(z) \rightarrow 0, \quad z \hat{\pi}^{\prime}(z) \rightarrow 0, \quad z \rightarrow \infty
$$

In addition,

$$
\hat{c}(z)>c_{0} \quad \text { for } z \geq 1 \text { if } \quad p<1, \quad \text { and } \quad \hat{c}(1)<c_{0} \text { if } \quad p>1 .
$$

The proof of Proposition 4.1 is deferred to Subsection 5.2.

Remark 4.1 Note that for any value of $\lambda$, no matter how large, the feedback control $\hat{\pi}$ is strictly positive. The investor makes a positive investment in the fund even for $\lambda>1$. The intuition is that even if fees are higher than 100\% of the achieved profit, after crossing the high-water mark and paying the high fee, the high-water mark is increased. The net profit of the investor grows in the long run with positive fund return $\alpha$ for any size of $\lambda>0$.

Remark 4.2 The optimal investment problem we consider allows for short-sales of the hedge-fund share (performance fees are still paid to the fund manager in this case). However, the optimal proportion $\hat{\pi}$ is positive, so this is the optimal portfolio selection even if short-sale constraints are imposed.

The solution $u$ cannot be computed in closed form, so we need to appeal to numerical methods, as in the examples in Subsection 4.3 below. However, closed form approximations of the solution $u$ (and, more importantly of the optimal controls) are possible. For example, one could consider the suboptimal controls $\pi_{0}$ and $c_{0}$ from the Merton proportion and solve the linear equation

$$
\left\{\begin{array}{l}
\mathcal{L}_{c_{0}, \theta_{0}} u=0 \\
-\lambda(1-p) u(1)+(1+\lambda) u^{\prime}(1)=0, \quad \lim _{z \rightarrow \infty} u(z)=\frac{1}{1-p} c_{0}^{-p} .
\end{array}\right.
$$

This can be done in closed form (with the help of Mathematica), and provides a subsolution of the HJB (26). However, the solution to (32) would be useful for our purposes only for some values of $\lambda$ (since we need the technical condition $u(1) \geq w_{*}$ ), so we still need to construct the subsolution (37) in Subsection 5.1 below for the general case. Taking also into account that solving (32) involves hypergeometric functions, in order to keep the presentation simple we leave this computation aside for future work on the the asymptotic analysis as $\lambda \searrow 0$, in the spirit of [17] and [10]. The closed form solution of (32) will play a central role in the asymptotic analysis. 


\subsection{Certainty Equivalent Analysis}

We evaluate the quantitative impact of paying profit share fee $\lambda$ on the initial wealth of the investor. The size of the value function does not provide any intuitive interpretation. A useful method is to compute the so-called certainty equivalent wealth. By definition, the certainty equivalent is such a size of initial bankroll $\tilde{x}$ that the agent would be indifferent between $\tilde{x}$ when paying zero fees, and wealth $x$ when paying profit-share fees $\lambda$, all other parameters being the same.

From (22) we infer the proper transformation by equating $v_{0}(\tilde{x})$ and $v(x, n)=x^{1-p} u(z)$. We solve for the quantity

$$
\frac{\tilde{x}(z)}{x}=\left(\frac{u(z)}{u_{0}}\right)^{\frac{1}{1-p}}=\left((1-p) c_{0}^{p} u(z)\right)^{\frac{1}{1-p}} z, \quad z \geq 1,
$$

which is the relative amount of wealth needed to achieve the same utility if no fee is paid (which also quantifies the proportional loss of wealth).

It is also useful to evaluate the size of the proportional fee (percentage per year, as in Remark 2.2) that would cause the same loss in utility as the current high-watermark performance fee. More precisely, we want to find the certainty equivalent excess return $\tilde{\alpha}<\alpha$ so that the value function obtained by using $\tilde{\alpha}$ and no fee is equal to the value function when the return is $\alpha$ but the performance fee is paid.

Keeping all the other parameters the same, the value function for zero performance-fee corresponding to excess return $\tilde{\alpha}$ is given by

$$
\tilde{u}_{0}(\tilde{\alpha})=\frac{1}{1-p} \tilde{c}_{0}(\tilde{\alpha})^{-p}
$$

with

$$
\tilde{c}_{0}(\tilde{\alpha})=\frac{\beta}{p}-\frac{1-p}{2 p^{2}} \cdot \frac{\tilde{\alpha}^{2}}{\sigma^{2}} .
$$

Therefore, we are looking for the solution to the equation

$$
\tilde{u}_{0}(\tilde{\alpha}(z))=u(z)
$$

which is given by

$$
\tilde{\alpha}^{2}(z)=2 \sigma^{2} \frac{p^{2}}{1-p}\left(\frac{\beta}{p}-((1-p) u(z))^{-\frac{1}{p}}\right), \quad z \geq 1 .
$$

The relative size of the certainty equivalent excess return is therefore

$$
\frac{\tilde{\alpha}(z)}{\alpha}=\frac{\sqrt{2} \sigma p}{\alpha}\left(\frac{\frac{\beta}{p}-((1-p) u(z))^{-\frac{1}{p}}}{1-p}\right)^{\frac{1}{2}}, \quad z \geq 1 .
$$

\subsection{Numerical Examples}

To the best of our knowledge, there is no closed form solution for the problem at hand. In order to properly understand the impact of the high-watermark fee on the investor, we need to resort to numerics.

In the next few charts we exhibit numerical results for various values of the parameters $p, \beta, \alpha$, $\sigma$ and, most importantly, the size of the high-watemark fee $\lambda$. All quantities are actually compared to the corresponding values in the Merton case $\lambda=0$. More precisely, we present four graphs, each representing, respectively,

- The size of the relative optimal investment proportion $\hat{\theta}(z) / \theta_{0}, z \geq 1$.

- The size of the relative optimal consumption proportion $\hat{c}(z) / c_{0}, z \geq 1$. 
- The relative size of the certainty equivalent initial wealth (which means the proportions $\tilde{x}_{0}(z) / x, z \geq$ 1 , in $(33))$.

- The relative size of the certainty equivalent excess return (which means $\tilde{\alpha}(z) / \alpha, z \geq 1$, in (34))

We remind the reader that the values for zero fee are obtained for $z \nearrow \infty$. This means that all the relative quantities presented in the graphs below approach one as $z \nearrow \infty$. For each of the four graphs representing the quantities described above, several values of the parameters $p, \beta, \alpha, \sigma$, and $\lambda$ are considered in the following manner:

- First, the size of the volatility is fixed to $\sigma=30 \%$. This is actually not restrictive since a model with given $\alpha$ and $\sigma$ has an identical value function as a scaled model with return $k \alpha$ and standard deviation $k \sigma$, while the investment proportion scales by $1 / k$. Since we draw the graph for the relative investment proportion (compared to the Merton case), this would actually not change at all, even by scaling.

- After that, we fix the benchmark parameters

$$
p=3, \beta=5 \%, \alpha=10 \%, \lambda=20 \% \text {. }
$$

Each of the parameters $p, \beta, \alpha$ and $\lambda$ is then varied around the benchmark values, keeping all the other parameters identical.

Remark 4.3 1. For the numerical examples below we can see that the optimal investment proportion at the high-watermark level $\hat{\pi}(1)$ is greater than the Merton proportion $\pi_{0}$. The intuitive explanation for this feature is that the agent wants to play the "local time game" at the boundary. When making a high investment proportion for a short time the loss in value due to over-investment is small, while the agent is able to push the high-watermark a little bit extra and benefit from an increased high-water mark in the future. This additional increase in high-water mark can be also interpreted as hedging.

2. The numerical analysis indicates that we can find sufficiently high $\beta$ so that $\hat{\pi}(1)<\pi_{0}$ for any set of parameters (with $\lambda>0$ ). However, we conjecture that $\hat{\pi}(z)$ is strictly decreasing at $z=1$ where the effect of pushing the high-watermark prevails over time preferences. We further conjecture that for $\beta=0$ (no time preferences of the investor) the function $\hat{\pi}(z)$ is strictly decreasing for all $z \geq 1$.

Remark 4.4 From Proposition 2.2 we see that paying the high-watemark has the effect of reducing the wealth of the investor by a factor of $1+\lambda$ in the long-run. At the same time, the certainty equivalent relative excess return $\tilde{\alpha}(z) / \alpha$ is usually (for reasonable values of the parameters, as below) larger than $1 /(1+\lambda)$. The intuitive reason is that the fees are paid only when an additional profit is being made, and thus the fees "hurt less" from utility point of view. No fees are paid in states when the investor loses money which would be the most painful from the utility point of view. 


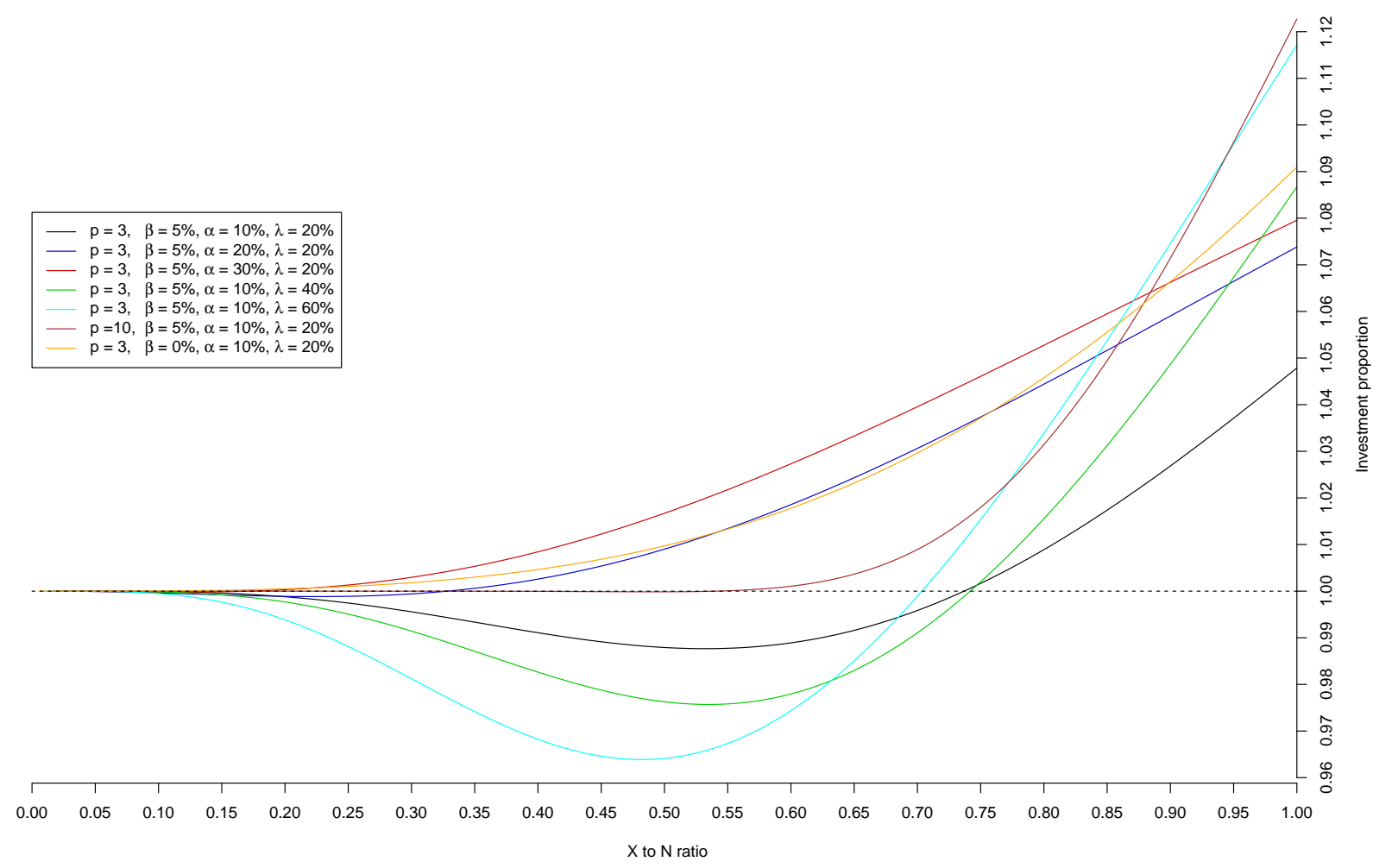

Consumption proportion relative to Merton consumption

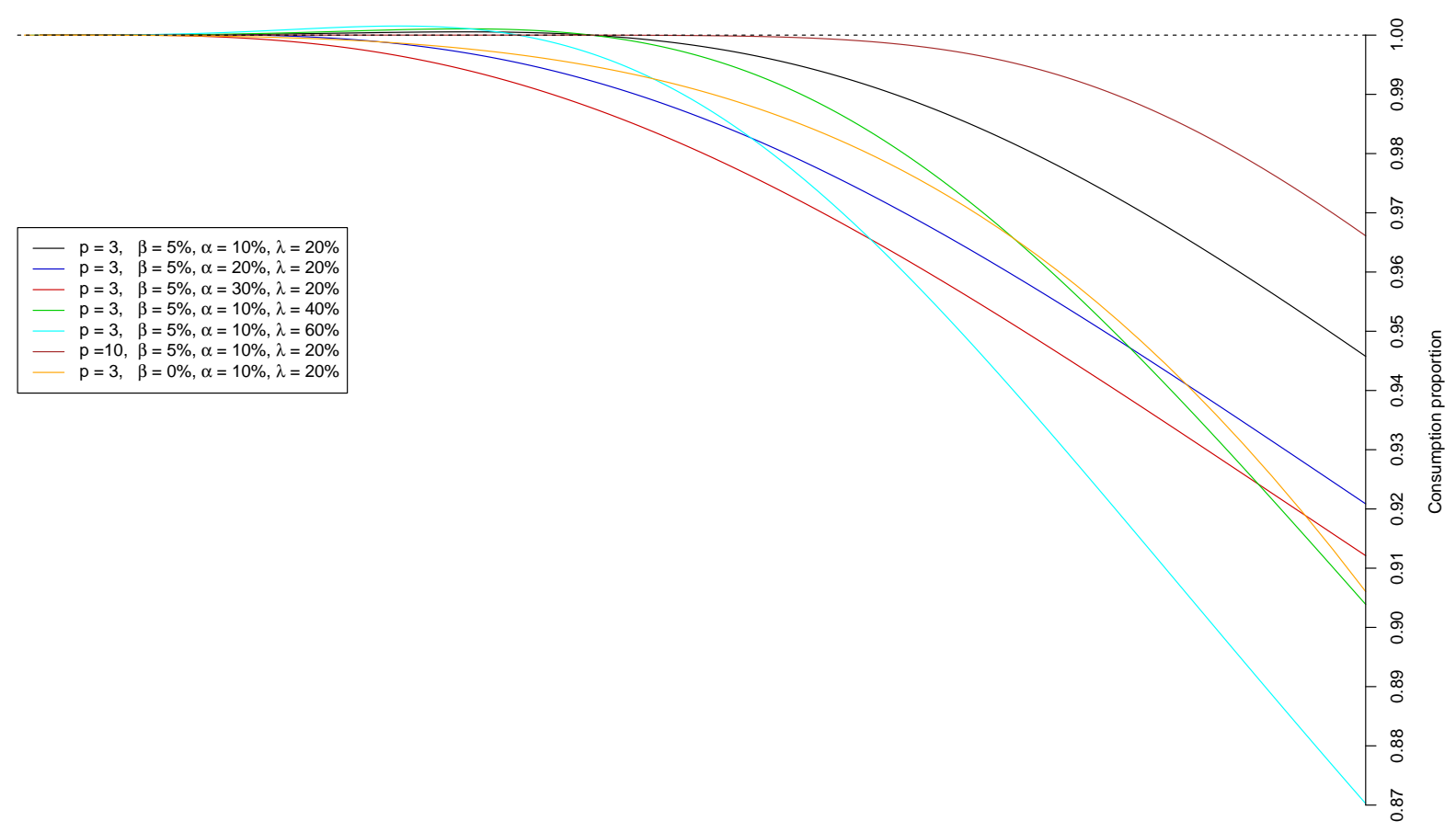

$\begin{array}{lllllllllllllllllllllllll}0.00 & 0.05 & 0.10 & 0.15 & 0.20 & 0.25 & 0.30 & 0.35 & 0.40 & 0.45 & 0.50 & 0.55 & 0.60 & 0.65 & 0.70 & 0.75 & 0.80 & 0.85 & 0.90 & 0.95 & 1.00\end{array}$ 

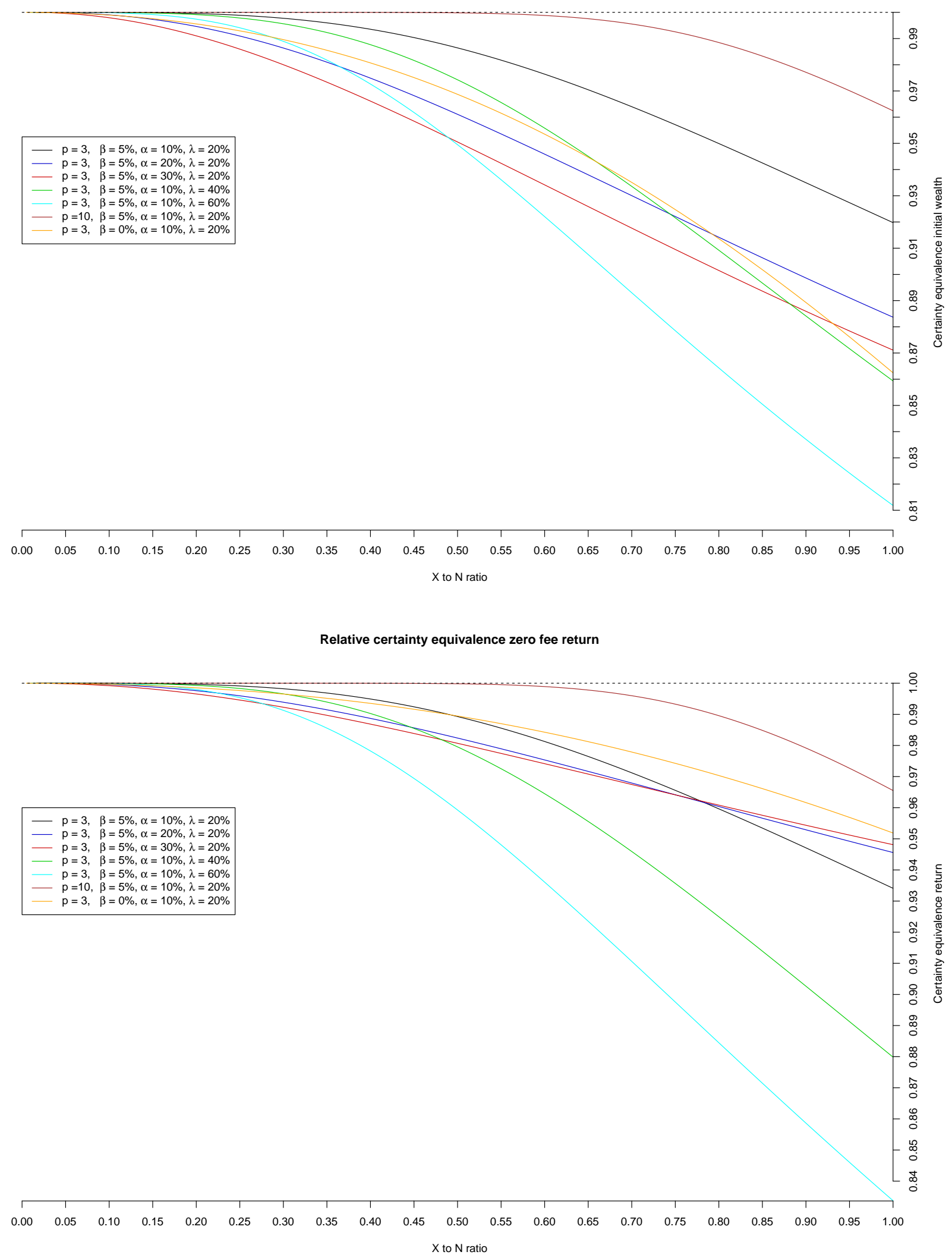


\section{Proofs}

The proof of Theorem 3.1 is split in between Subsections 5.1 and 5.2 below. In Subsection 5.1 we proof the existence of a viscosity solution using Perron's method, and then we upgrade its regularity in Subsection 5.2. We refer the reader to the seminal paper [3] for an introduction to the theory of viscosity solutions. We use here only the well-known notion of viscosity solution on an open domain, since boundary conditions can be interpreted in the classical sense. In order to keep the presentation more streamlined, we do not include the definition here.

\subsection{Existence of a viscosity solution}

We observe that the constant solution $u_{0}$ corresponding to the case $\lambda=0$ (defined in (23) in Remark 3.1 ) is actually a classical supersolution of the HJB (26), which reads

$$
\left\{\begin{array}{l}
\left.\sup _{c>0, \theta} \mathcal{L}_{c, \theta} u=-\beta u+\tilde{V}((1-p) u-(z-1) u)\right)-\frac{1}{2} \frac{\alpha^{2}}{\sigma^{2}} \frac{\left((1-p) u-z u^{\prime}\right)^{2}}{-p(1-p) u+2 p z u^{\prime}+z^{2} u^{\prime \prime}} \leq 0 \\
-\lambda(1-p) u(1)+(1+\lambda) u^{\prime}(1) \leq 0, \quad \lim _{z \rightarrow \infty} u(z) \geq \frac{1}{1-p} c_{0}^{-p} .
\end{array}\right.
$$

For technical reasons we also need a subsolution with certain properties. We remind the reader that the critical value $w_{*}$ was defined in $(27)$.

Proposition 5.1 There exists a value $z_{*} \in(1, \infty)$ and a function

$$
u_{s} \in C^{1}[1, \infty) \cap C^{2}\left(1, z_{*}\right] \cap C^{2}\left[z_{*}, \infty\right)
$$

such that

$$
w_{*} \leq u_{s} \leq u_{0}
$$

and $u_{s}$ is a strict viscosity subsolution for (26). More precisely, $u_{s}$ satisfies

$$
\sup _{c>0, \theta} \mathcal{L}_{c, \theta} u_{s}>0
$$

in the viscosity sense on $(1, \infty)$, and

$$
-\lambda(1-p) u_{s}(1)+(1+\lambda) u_{s}^{\prime}(1)>0, \quad \lim _{z \rightarrow \infty} u_{s}(z)<\frac{1}{1-p} c_{0}^{-p} .
$$

Proof: For

$$
\frac{\lambda}{1+\lambda}(1-p) w_{*}<a<(1-p) w_{*}
$$

we consider the function

$$
u_{s}(z)=\left\{\begin{array}{l}
w_{*}+a(z-1)-\frac{2 a}{1+\varepsilon}(z-1)^{1+\varepsilon}, \quad 1 \leq z \leq 1+\left(\frac{1}{2}\right)^{\frac{1}{\varepsilon}} \\
w_{*}+a \frac{\varepsilon}{1+\varepsilon}\left(\frac{1}{2}\right)^{\frac{1}{\varepsilon}}, \quad z \geq 1+\left(\frac{1}{2}\right)^{\frac{1}{\varepsilon}}
\end{array}\right.
$$

Since

$$
\begin{array}{r}
\left.\tilde{V}\left((1-p)\left(w_{*}+a(z-1)-\frac{2 a}{1+\varepsilon}(z-1)^{1+\varepsilon}\right)-a\left((z-1)-2(z-1)^{1+\varepsilon}\right)\right) \geq \tilde{V}\left((1-p) w_{*}\right)\right)+ \\
\left.+\tilde{V}^{\prime}\left((1-p) w_{*}\right)\right)\left((1-p)\left(a(z-1)-\frac{2 a}{1+\varepsilon}(z-1)^{1+\varepsilon}\right)-a\left((z-1)-2(z-1)^{1+\varepsilon}\right)\right)
\end{array}
$$


we notice that

$$
\begin{aligned}
& \sup _{c, \pi} \mathcal{L}_{c, \pi} u_{s}(z) \geq-\beta\left(a(z-1)-\frac{2 a}{1+\varepsilon}(z-1)^{1+\varepsilon}\right)+ \\
& \left.+\tilde{V}^{\prime}\left((1-p) w_{*}\right)\right)\left((1-p)\left(a(z-1)-\frac{2 a}{1+\varepsilon}(z-1)^{1+\varepsilon}\right)-a\left((z-1)-2(z-1)^{1+\varepsilon}\right)\right) \\
& +\frac{1}{2} \frac{\alpha^{2}}{\sigma^{2}} \frac{\left(\left((1-p) w_{*}\right) \wedge\left((1-p)\left(w_{*}+\frac{a \varepsilon}{1+\varepsilon}\left(\frac{1}{2}\right)^{\frac{1}{\varepsilon}}\right)\right)-\left(1+\left(\frac{1}{2}\right)^{\frac{1}{\varepsilon}}\right) a\right)^{2}}{p\left(\left((1-p) w_{*}\right) \vee\left((1-p)\left(w_{*}+\frac{a \varepsilon}{1+\varepsilon}\left(\frac{1}{2}\right)^{\frac{1}{\varepsilon}}\right)\right)\right)+\left(1+\left(\frac{1}{2}\right)^{\frac{1}{\varepsilon}}\right)^{2} 2 a \varepsilon(z-1)^{-(1-\varepsilon)}}
\end{aligned}
$$

Since $a<(1-p) w_{*}$, it turns out that we can choose some constants $C$ and $D$ as well as $F, G, H>0$, independent of small $\varepsilon$ such that, for all $1<z \leq 1+\left(\frac{1}{2}\right)^{\frac{1}{\varepsilon}}$, we have

$$
\begin{aligned}
\sup _{c, \pi} \mathcal{L}_{c, \pi} u_{s}(z) \geq \frac{F}{G+H \varepsilon(z-1)^{-(1-\varepsilon)}}-C(z-1)-D(z-1)^{1+\varepsilon} \geq \\
\geq \frac{F}{G+H \varepsilon(z-1)^{-(1-\varepsilon)}}-|C|(z-1)-|D|(z-1)^{1+\varepsilon} .
\end{aligned}
$$

We now see that

$$
\begin{aligned}
& \left(G+H \varepsilon(z-1)^{-(1-\varepsilon)}\right)\left(|C|(z-1)+|D|(z-1)^{1+\varepsilon}\right)= \\
& =\left(G(z-1)^{1-\varepsilon}+H \varepsilon\right)\left(|C|(z-1)^{\varepsilon}+|D|(z-1)^{2 \varepsilon}\right) \leq \\
& \quad \leq\left(2 G\left(\frac{1}{2}\right)^{\frac{1}{\varepsilon}}+H \varepsilon\right)\left(\frac{1}{2}|C|+\frac{1}{4}|D|\right)<F, \text { for all } 1<z \leq 1+\left(\frac{1}{2}\right)^{\frac{1}{\varepsilon}},
\end{aligned}
$$

if $\varepsilon$ is small enough. Therefore, for such an $\varepsilon$ we will have

$$
\sup _{c, \pi} \mathcal{L}_{c, \pi} u_{s}(z)>0, \quad 1<z \leq 1+\left(\frac{1}{2}\right)^{\frac{1}{\varepsilon}} .
$$

Since $u_{s}$ is constant for $z \geq 1+\left(\frac{1}{2}\right)^{\frac{1}{\varepsilon}}$ and is extended to be $C^{1}$ we obtain

$$
\sup _{c, \pi} \mathcal{L}_{c, \pi} u_{s}(z)>0, \quad z>1
$$

in the viscosity sense, and actually in the classical sense for any $z \neq z_{*} \triangleq(1 / 2)^{1 / \varepsilon}$. $\diamond$

We now construct a viscosity solution of the HJB using Perron's method. More precisely, we denote by $\mathcal{S}$ the set of functions

$$
h:[1, \infty) \rightarrow \mathbb{R},
$$

which satisfy the following properties

1. $h$ is continuous on $[1, \infty)$

2. for fixed $n$, the function $x \rightarrow x^{1-p} h(n / x)$ is concave and nondecreasing in $x \in(0, n]$

3. $h$ is a viscosity supersolution of the (HJB) equation on the open interval $(1, \infty)$

4. $-\lambda(1-p) h(1)+(1+\lambda) h^{\prime}(1) \leq 0$

5. $u_{s} \leq h \leq u_{0}$ 
Theorem 5.1 Define

$$
u \triangleq \inf \{h, \quad h \in \mathcal{S}\} .
$$

Then, $u_{s} \leq u \leq u_{0}$ is continuous on $[1, \infty)$, is a viscosity solution of the (HJB) on the open interval $(1, \infty)$, and satisfies $-\lambda(1-p) u(1)+(1+\lambda) u^{\prime}(1)=0$. In addition, for fixed $n$, the function $x \rightarrow$ $x^{1-p} u(n / x)$ is concave and nondecreasing in $x \in(0, n]$, and $u(1)>w_{*}$.

Proof: The proof follows the ideas of the proof of Theorem 4.1 in [3], with the necessary technical modifications to take into account the boundary condition at $z=1$ and to keep track of the convexity properties.

1. By construction, as an infimum of concave nondecreasing functions, we have that for fixed $n$, the function $x \rightarrow x^{1-p} u(n / x)$ is concave and nondecreasing in $x \in(0, n]$.

2. Since $x \rightarrow x^{1-p} u(n / x)$ is concave and nondecreasing in $x \in(0, n]$ we conclude that $x \rightarrow$ $x^{1-p} u(n / x)$ is continuous on $(0, n]$, which translates to $u$ is continuous on $[1, \infty)$

3. Assume a $C^{2}$ function $\varphi$ touches $u$ from below at an interior point $z \in(1, \infty)$. For fixed $c, \theta$, each $h \in \mathcal{S}$ is a viscosity supersolution of $\mathcal{L}_{c, \theta} h \leq 0$, so taking the inf over $h \in \mathcal{S}$ we still get a supersolution, according to Lemma 4.2 in [3]. In other words, $\left(\mathcal{L}_{c, \theta} \varphi\right)(z) \leq 0$, and then we can take the sup over $(c, \theta)$ to get that $u$ is a supersolution of the (HJB).

We would like to mention that Lemma 4.2 in [3] works without the fundamental monotonicity condition $(0.1)$ in [3].

4. By construction, $u_{s} \leq u \leq u_{0}$.

5. (The boundary condition.) For each $h \in \mathcal{S}$, we have

$$
h^{\prime}(1) \leq \frac{\lambda}{1+\lambda}(1-p) h(1)
$$

which translates in terms of $g(x, n) \triangleq x^{1-p} h(n / x)$ as

$$
g_{x}(1,1)=(1-p) h(1)-h^{\prime}(1) \geq \frac{1}{1+\lambda}(1-p) h(1) .
$$

Taking into account the concavity of $g(x, n)$ in $x \in(0, n]$, this is equivalent to

$$
g(x, 1)=x^{1-p} h(m / x) \leq \frac{1}{1+\lambda}(1-p) h(1)(x-1)+h(1), \quad 0<x \leq 1 .
$$

Since (38) holds for each $h \in \mathcal{S}$ the same thing will hold for the infimum, which means that $u$ satisfies (38), which reads:

$$
u^{\prime}(1) \leq \frac{\lambda}{1+\lambda}(1-p) u(1)
$$

Assume now that the inequality above is strict. Since $u_{s}^{\prime}(1)=a>\frac{\lambda}{1+\lambda}(1-p) u_{s}(1)$ and $u \geq u_{s}$, this rules out the possibility that $u(1)=u_{s}(1)$, so we have $u(1)>u_{s}(1)=w_{*}$. This implies

$$
-\beta u(1)+\tilde{V}((1-p) u(1))<0 .
$$

In addition, we have

$$
(1-p) u(1)-u^{\prime}(1)>\frac{1}{1+\lambda}(1-p) u(1) \geq 0 .
$$

Therefore, we can find a number $a^{\prime}$ such that

$$
u^{\prime}(1)<a^{\prime}<\frac{\lambda}{1+\lambda}(1-p) u(1),
$$


and a very large $b>0$ such that the function

$$
\psi(z)=u(1)+a^{\prime}(z-1)-\frac{1}{2} b(z-1)^{2}
$$

is, in a small neighborhood $(1,1+\delta)$ of $z=1$, a classical supersolution. In addition, it satisfies $\psi(1)^{\prime}=a^{\prime}<\frac{\lambda}{1+\lambda} \psi(1)$. Thus, if $\delta$ is small enough, we have that $u(z)<\psi(z)$ on $(1,1+\delta)$, and

$$
(1-p) \psi(z)-z \psi^{\prime}(z)>0, \quad-p(1-p) \psi(z)+2 p z \psi^{\prime}(z)+z^{2} \psi^{\prime \prime}(z)<0, \quad z \in[1,1+\delta] .
$$

Now, for a very small $\varepsilon$, at least as small as

$$
\varepsilon_{0} \triangleq \min _{z \in\left[1+\frac{\delta}{2}, 1+\delta\right]} \psi(z)-u(z)
$$

but possibly even smaller, we have that the function

$$
\tilde{u}(z) \triangleq\left\{\begin{array}{l}
\min \{u(z), \psi(z)-\varepsilon\}, \quad z \in[1,1+\delta] \\
u(z), \quad z \in[1+\delta, \infty)
\end{array}\right.
$$

is actually an element of $\mathcal{S}$, contradicting the assumption that $u$ is the infimum over $\mathcal{S}$.

6. Let us show that $u$ is a viscosity subsolution. We first start by making the following simple observation on the function $u$ : By construction, the function $(x, n) \rightarrow x^{1-p} u(n / x)$ is concave in $x \in[0, n]$. Since

$$
v_{x}(x-, n)=\left((1-p) u(z)-z u^{\prime}(z+)\right) \cdot x^{-p}, \quad v_{x}(x+, n)=\left((1-p) u(z)-z u^{\prime}(z-)\right) \cdot x^{-p},
$$

we obtain

$$
(1-p) u(z)-z u^{\prime}(z+) \geq(1-p) u(z)-z u^{\prime}(z-), \quad z>1,
$$

which of course means that $u^{\prime}(z-) \geq u^{\prime}(z+)$ for $z>1$. Assume, by contradiction, that for some $z_{0}>1$ we have $\left((1-p) u\left(z_{0}\right)-z u^{\prime}\left(z_{0}-\right)\right)=0$. Then, we have that $v_{x}\left(\frac{1}{z_{0}}+, 1\right)=0$, which, together with the fact that $x \rightarrow v(x, 1)$ is concave and nondecreasing for $x \in\left[\frac{1}{z_{0}}, 1\right]$, shows that actually $v(x, 1)=v(1,1)$ for $x \in\left[\frac{1}{z_{0}}, 1\right]$. This means that

$$
v_{x}(1,1)=(1-p) u(1)-u^{\prime}(1)=0
$$

which is a contradiction to the boundary condition

$$
u^{\prime}(1)=\frac{\lambda}{1+\lambda}(1-p) u(1)
$$

Therefore, for any $z>1$ we have

$$
(1-p) u(z)-z u^{\prime}(z+) \geq(1-p) u(z)-z u^{\prime}(z-)>0 .
$$

Assume now that a $C^{2}$ function $\varphi$ touches $u$ from above at some interior point $z \in(1, \infty)$. If $u(z)=u_{s}(z)$ we can use the test function $u_{s}$ (which is a strict subsolution) for the supersolution $u$ to obtain a contradicition. The contradiction argument works even if $z=(1 / 2)^{1 / \varepsilon}$ is the only exceptional point where $u_{s}$ is not $C^{2}$. Therefore, $u(z)>u_{s}(z)$. From (39) we can easily conclude that

$$
(1-p) \varphi(z)-z \varphi^{\prime}(z)>0 .
$$

Assume now, that $u$ does not satisfy the sub-solution property, which translates to

$$
\sup _{c, \theta}\left(\mathcal{L}_{(c, \theta)} \varphi\right)(z)<0 .
$$


Since $(1-p) \varphi(z)-z \varphi^{\prime}(z)>0$ we can conclude that

$$
-p(1-p) \varphi(z)+2 p z \varphi^{\prime}(z)+z^{2} \varphi^{\prime \prime}(z)<0, \quad(1-p) \varphi(z)-(z-1) \varphi^{\prime}(z)>0,
$$

and

$$
\left.-\beta \varphi(z)+\tilde{V}\left((1-p) \varphi(z)-(z-1) \varphi^{\prime}(z)\right)\right)-\frac{1}{2} \frac{\alpha^{2}}{\sigma^{2}} \frac{\left((1-p) \varphi(z)-z \varphi^{\prime}(z)\right)^{2}}{-p(1-p) \varphi(z)+2 p z \varphi^{\prime}(z)+z^{2} \varphi^{\prime \prime}(z)}<0 .
$$

Actually, relations (40) and (41) hold in a small neighborhood $(z-\delta, z+\delta)$ of $z$, not just at $z$. Furthermore, for $\eta>0$ small enough, relations (40) and (41) hold $\operatorname{in}(z-\delta, z+\delta)$ for the slightly modified function

$$
\psi(w) \triangleq \varphi(w)+\frac{\eta}{2}(w-z)^{2} .
$$

Considering an even a smaller $\delta$ we have that $u(w)<\psi(w)$, for $w \in[z-\delta, z+\delta]$ if $w \neq z$. Now, for a $\varepsilon$ small enough, which means at least as small as

$$
\varepsilon_{0} \triangleq \min _{\frac{\delta}{2} \leq|w-z| \leq \delta} \psi(w)-u(w)
$$

but maybe much smaller, we define the function

$$
\tilde{u}(w) \triangleq\left\{\begin{array}{l}
\min \{u(w), \psi(w)-\varepsilon\}, \quad w \in[z-\delta, z+\delta] \\
u(w), \quad w \notin[z-\delta, z+\delta]
\end{array}\right.
$$

We note that, if $\varepsilon$ is indeed small enough, we have that $\tilde{u} \in \mathcal{S}$ and $\tilde{u}$ is strictly smaller than $u$ (around $z)$, which is a contradiction. $\diamond$

\subsection{Smoothness of the viscosity solution}

Theorem 5.2 The function $u$ in Theorem 5.1 is $C^{2}$ on $[1, \infty)$, and satisfies the conditions

$$
-p(1-p) u+2 p z u^{\prime}+z^{2} u^{\prime \prime}<0, \quad(1-p) u-(z-1) u^{\prime}>0, \quad(1-p) u-z u^{\prime}>0, \quad z \geq 1 .
$$

Moreover it is a solution of the equation

$$
\left\{\begin{array}{l}
\left.\sup _{c>0, \theta} \mathcal{L}_{c, \theta} u=-\beta u+\tilde{V}((1-p) u-(z-1) u)\right)-\frac{1}{2} \frac{\alpha^{2}}{\sigma^{2}} \frac{\left((1-p) u-z u^{\prime}\right)^{2}}{-p(1-p) u+2 p z u^{\prime}+z^{2} u^{\prime \prime}}=0, \quad z \geq 1 \\
-\lambda(1-p) u(1)+(1+\lambda) u^{\prime}(1)=0 .
\end{array}\right.
$$

Proof: First, we remind the reader that the dual function $\tilde{V}(y)$ is defined for all values of $y$, not only for $y>0$. More precisely

$$
\tilde{V}(y)=\left\{\begin{array}{l}
\frac{p}{1-p} y^{\frac{p-1}{p}}, y>0 \\
+\infty, \quad y \leq 0
\end{array} \quad \text { for } p<1, \quad \tilde{V}(y)=\left\{\begin{array}{l}
\frac{p}{1-p} y^{\frac{p-1}{p}}, y \geq 0 \\
+\infty, \quad y<0
\end{array} \quad \text { for } p>1\right.\right.
$$

Let $z_{0}>1$ such that $u^{\prime}\left(z_{0}-\right)>u^{\prime}\left(z_{0}+\right)$. For each $u^{\prime}\left(z_{0}+\right)<a<u^{\prime}\left(z_{0}-\right)$ and $b>0$ large enough we use the function

$$
\psi(z) \triangleq u\left(z_{0}\right)+a\left(z-z_{0}\right)-\frac{1}{2} b\left(z-z_{0}\right)^{2}
$$

as a test function for the viscosity subsolution property, so

$$
\sup _{c, \theta}\left\{-\beta u\left(z_{0}\right)+\frac{c^{1-p}}{1-p}+(\theta \alpha-c)\left((1-p) u\left(z_{0}\right)-z_{0} a\right)-c a+\frac{1}{2} \theta^{2} \sigma^{2}\left(-p(1-p) u\left(z_{0}\right)+2 p z_{0} a+z_{0}^{2}(-b)\right)\right\} \geq 0 .
$$


Since $(1-p) u\left(z_{0}\right)-z_{0} a>(1-p) u\left(z_{0}\right)-z_{0} u^{\prime}\left(z_{0}-\right)>0$ the above equation can be rewritten as

$$
\left.-\beta u\left(z_{0}\right)+\tilde{V}\left((1-p) u\left(z_{0}\right)-\left(z_{0}-1\right) a\right)\right)-\frac{1}{2} \frac{\alpha^{2}}{\sigma^{2}} \frac{\left((1-p) u\left(z_{0}\right)-z_{0} a\right)^{2}}{p(1-p) u\left(z_{0}\right)+2 p z_{0} a-b z_{0}^{2}} \geq 0 .
$$

Letting $b \rightarrow \infty$, we obtain

$$
\left.-\beta u\left(z_{0}\right)+\tilde{V}\left((1-p) u\left(z_{0}\right)-\left(z_{0}-1\right) a\right)\right) \geq 0, \quad a \in\left(u^{\prime}\left(z_{0}+\right), u^{\prime}\left(z_{0}-\right)\right),
$$

so

$$
\left.-\beta u\left(z_{0}\right)+\tilde{V}\left((1-p) u\left(z_{0}\right)-\left(z_{0}-1\right) u^{\prime}\left(z_{0}-\right)\right)\right)>0 .
$$

Since $u^{\prime}(z-)$ is left continuous, and the function $u$ is two times differentiable on a dense set $\mathcal{D} \subset(1, \infty)$ by convexity, there exists $1<z<z_{0}$ such that $z \in \mathcal{D}$, and

$$
\left.-\beta u(z)+\tilde{V}\left((1-p) u(z)-(z-1) u^{\prime}(z)\right)\right) \geq 0 .
$$

However, this would contradict the viscosity supersolution property at $z$, which reads

$$
\begin{aligned}
-\beta u(z) & \left.+\tilde{V}\left((1-p) u(z)-(z-1) u^{\prime}(z)\right)\right)+ \\
& +\sup _{\theta}\left\{\theta \alpha\left((1-p) u(z)-z u^{\prime}(z)\right)+\frac{1}{2} \theta^{2} \sigma^{2}\left(-p(1-p) u(z)+2 p z u^{\prime}(z)-z^{2} u^{\prime \prime}(z)\right)\right\} \leq 0,
\end{aligned}
$$

since we have shown that $(1-p) u(z)-z u^{\prime}(z)>0$ (actually all left and right derivatives like that are positive). We obtained a contradiction, so we have proved that

$$
u^{\prime}\left(z_{0}-\right)=u^{\prime}\left(z_{0}+\right), \quad \text { for all } z_{0}>1
$$

In other words, $u^{\prime}$ is well defined and continuous on $[1, \infty)$. In addition, $(1-p) u(z)-z u^{\prime}(z)>0$ for $z \geq 1$. Applying again the viscosity solution property at a point where $u$ is two times differentiable we obtain

$$
\left.-\beta u(z)+\tilde{V}\left((1-p) u(z)-(z-1) u^{\prime}(z)\right)\right)<0, \quad z \in \mathcal{D},
$$

and

$$
z^{2} u^{\prime \prime}+2 p z u^{\prime}-p(1-p) u=\frac{\alpha^{2}}{2 \sigma^{2}} \frac{\left((1-p) u-z u^{\prime}\right)^{2}}{-\beta u+\tilde{V}\left((1-p) u-(z-1) u^{\prime}\right)}<0, \quad z \in \mathcal{D} .
$$

Using continuity and the density of $\mathcal{D}$, we get

$$
\left.f(z) \triangleq \beta u(z)-\tilde{V}\left((1-p) u(z)-(z-1) u^{\prime}(z)\right)\right) \geq 0, \quad z \geq 1 .
$$

Taking into account the bounds we have on $u$, we can use (44) to obtain

$$
\left.(1-p) u(z)-(z-1) u^{\prime}(z)\right)>0, \quad z \geq 1 .
$$

The function $f$ defined in (44) is continuous. We claim that on any open interval $(a, b) \subset[1, \infty)$ such that $f(z)>0$ on $[a, b]$, we have that $u$ is $C^{2}$ and satisfies the equation. The claim is easy to prove, noting that $u$ is a viscosity solution of the equation

$$
u-u^{\prime \prime}=u-\frac{1}{z^{2}}\left[-\left(2 p z u^{\prime}-p(1-p)\right)+\frac{\alpha^{2}}{2 \sigma^{2}} \frac{\left((1-p) u-z u^{\prime}\right)^{2}}{-\beta u+\tilde{V}\left((1-p) u-(z-1) u^{\prime}\right)}\right], \quad z \in(a, b),
$$

and the right hand side is continuous up to the boundaries of $(a, b)$. Comparing to the classical solution of this equation with the very same right hand side and Dirichlet boundary conditions at $a$ and $b$, we finish the proof of the claim. We would like to point out that the comparison argument between 
the viscosity solution and the classical solution is straightforward, and does not involve any doubling argument.

It, therefore, remains to show that $f(z)>0$ for all $z \geq 1$. According to Theorem 5.1 we have $u(1)>w_{*}$, so $f(1)>0$. Continuity implies that $f>0$ in a small neighborhood to the right of $z=1$. Assume that the function $f$ vanishes for some $z>1$. Let some $b>1$ be the smallest $z$ such that the function vanishes at $z$. This means that $1<b$ and $f(b)=0$, and $f(z)>0$ for $z \in(1, b)$. On $(1, b)$ we know that $u$ is $C^{2}$ and the equation is satisfied, so we have

$$
\frac{f(z)-f(b)}{z-b}=b u^{\prime}(\eta)-\tilde{V}^{\prime}\left((1-p) u(\eta)-(\eta-1) u^{\prime}(\eta)\right)\left\{(1-p) u^{\prime}(\eta)-u^{\prime}(\eta)-(\eta-1) u^{\prime \prime}(\eta)\right\} .
$$

From the fact that the equation is satisfied on $(a, b)$ and $f(b)=0$, we have that

$$
\lim _{\eta \succ b} u^{\prime \prime}(\eta)=-\infty
$$

so

$$
\lim _{z \nearrow b} \frac{f(z)-f(b)}{z-b}=+\infty
$$

since $\tilde{V}^{\prime}$ is continuous on $(0, \infty)$ and $\tilde{V}^{\prime}\left((1-p) u(b)-(b-1) u^{\prime}(b)\right)<0$. However, this is impossible, since

$$
\frac{f(z)-f(b)}{z-b} \leq 0
$$

Therefore $u$ is $C^{2}$ in $(1, \infty)$ and satisfies the (HJB). Since $u(1)>w_{*}$ which reads $f(1)>0$, for $f$ defined in (44), we can use continuity and pass to the limit in the (HJB) for $z \searrow 1$ to conclude that actually $u$ is $C^{2}$ in $[1, \infty)$ and the (HJB) is satisfied at the boundary as well.

Lemma 5.1 The function $u$ is strictly increasing on $[1, \infty)$ and

$$
\lim _{z \rightarrow \infty} u(z)=u_{0}, \lim _{z \rightarrow \infty} z u^{\prime}(z)=0, \lim _{z \rightarrow \infty} z^{2} u^{\prime \prime}(z)=0 .
$$

Proof: Assume that the function $u$ admits an interior minimum point $z_{0}$ over an interval $[a, b] \subset[1, \infty)$. Then $u^{\prime}\left(z_{0}\right)=0$ and $u^{\prime \prime}\left(z_{0}\right) \geq 0$. Replacing this into the equation (26) we get

$$
\left.-\beta u\left(z_{0}\right)+\tilde{V}\left((1-p) u\left(z_{0}\right)-\left(z_{0}-1\right) u^{\prime}\left(z_{0}\right)\right)\right)-\frac{1}{2} \frac{\alpha^{2}}{\sigma^{2}} \frac{\left((1-p) u\left(z_{0}\right)-z_{0} u^{\prime}\left(z_{0}\right)\right)^{2}}{-p(1-p) u\left(z_{0}\right)+2 p z_{0} u^{\prime}\left(z_{0}\right)+z_{0}^{2} u^{\prime \prime}\left(z_{0}\right)}=0,
$$

so

$$
\left.\beta u\left(z_{0}\right)-\tilde{V}\left((1-p) u\left(z_{0}\right)\right)\right)=\frac{1}{2} \frac{\alpha^{2}}{\sigma^{2}} \frac{\left((1-p) u\left(z_{0}\right)\right)^{2}}{p(1-p) u\left(z_{0}\right)-z_{0}^{2} u^{\prime \prime}\left(z_{0}\right)} \geq \frac{1}{2} \frac{\alpha^{2}}{\sigma^{2}} \frac{1-p}{p} u\left(z_{0}\right) .
$$

This can be rewritten as

$$
\left.\left(\beta-\frac{1}{2} \frac{\alpha^{2}}{\sigma^{2}} \frac{1-p}{p}\right) u\left(z_{0}\right) \geq \tilde{V}\left((1-p) u\left(z_{0}\right)\right)\right)
$$

Since

$$
\left.\beta-\frac{1}{2} \frac{\alpha^{2}}{\sigma^{2}} \frac{1-p}{p}>0, \text { and } \quad\left(\beta-\frac{1}{2} \frac{\alpha^{2}}{\sigma^{2}} \frac{1-p}{p}\right) u_{0}=\tilde{V}\left((1-p) u_{0}\right)\right)
$$

we obtain that $u\left(z_{0}\right) \geq u_{0}$ so $u\left(z_{0}\right)=u_{0}$. Now, we have two distinct classical global solutions $u, u_{0}$ of the differential equation

$$
z^{2} u^{\prime \prime}+2 p z u^{\prime}-p(1-p) u=\frac{\alpha^{2}}{2 \sigma^{2}} \frac{\left((1-p) u-z u^{\prime}\right)^{2}}{-\beta u+\tilde{V}\left((1-p) u-(z-1) u^{\prime}\right)}<0, \quad z>1,
$$


satisfying $u\left(z_{0}\right)=u_{0}\left(z_{0}\right)$ and $u^{\prime}\left(z_{0}\right)=u_{0}^{\prime}\left(z_{0}\right)=0$, which is a contradiction. This means that $u$ can only attain its minimum at the boundaries of an interval, which precludes the possibility

$$
\liminf _{z \rightarrow \infty} u(z)<\limsup _{z \rightarrow \infty} u(z) .
$$

Since $u$ is bounded we conclude that there exists

$$
u(\infty) \triangleq \lim _{z \rightarrow \infty} u(z) \in(-\infty, \infty)
$$

Now, since $u$ is bounded and $u^{\prime}$ is continuous we conclude, by contradiction, that there exist a sequence $z_{n} \nearrow \infty$ such that

$$
z_{n} u^{\prime}\left(z_{n}\right) \rightarrow 0, \quad n \rightarrow \infty .
$$

(Otherwise we would have $z\left|u^{\prime}(z)\right| \geq \varepsilon$ for some $\varepsilon$ for large $z$, which contradicts boundedness). Denote now

$$
0 \geq A:=\liminf _{z \rightarrow \infty} z u^{\prime}(z) \leq \limsup _{z \rightarrow \infty} z u^{\prime}(z)=: B \geq 0 .
$$

For fixed $C \in \mathbb{R}$, denote by

$$
f_{C}(z)=C u+z u^{\prime}, z \geq 1
$$

The function $f_{C}$ is continuous and

$$
\liminf _{z \rightarrow \infty} f_{C}(z)=C u(\infty)+A \leq C u(\infty)+B=\limsup f_{z \rightarrow \infty} f_{C}(z)
$$

Assume, by contradiction, that $0<B \leq \infty$. Since $\lim _{n \rightarrow \infty} f_{C}\left(z_{n}\right)=C u(\infty)<C u(\infty)+B$, then we can choose the points $\eta_{n} \in\left(z_{n}, z_{n+1}\right)$ (interior points, and eventually for a subsequence $n_{k}$ rather than for each $n$ ) for which $f_{C}$ attains the max on $\left[z_{n}, z_{n+1}\right]$ such that $f_{C}\left(\eta_{n}\right) \rightarrow C u(\infty)+B$, which is the same as $\eta_{n} u^{\prime}\left(\eta_{n}\right) \rightarrow B$. Since $f_{C}$ attains the interior maximum on on interval at $\eta_{n}$, we have $f_{C}^{\prime}\left(\eta_{n}\right)=(1+C) u^{\prime}\left(\eta_{n}\right)+\eta_{n} u^{\prime \prime}\left(\eta_{n}\right)=0$. Now, we know that

$$
-p(1-p) u\left(\eta_{n}\right)+2 p \eta_{n} u^{\prime}\left(\eta_{n}\right)+\eta_{n}^{2} u^{\prime \prime}\left(\eta_{n}\right) \leq 0
$$

or

$$
-p(1-p) u\left(\eta_{n}\right)+(2 p-1-C) \eta_{n} u^{\prime}\left(\eta_{n}\right) \leq 0 .
$$

Passing to the limit, we obtain that

$$
-p(1-p) u(\infty)+(2 p-1-C) B \leq 0,
$$

for each $C \in \mathbb{R}$, which means that $B=0$. Similarly, we obtain $A=0$ so $z u^{\prime}(z) \rightarrow 0$. Now, since $z u^{\prime}$ is bounded and $\left(z u^{\prime}\right)^{\prime}$ is continuous we conclude, by contradiction, that there exist a sequence $z_{n} \nearrow \infty$ such that

$$
\left(z_{n}\right)^{2} u^{\prime \prime}\left(z_{n}\right) \rightarrow 0, \quad n \rightarrow \infty .
$$

Passing to the limit along $z_{n}$ 's in the HJB, we obtain

$$
\beta u(\infty)-\tilde{V}((1-p) u(\infty)))=\frac{1}{2} \frac{\alpha^{2}}{\sigma^{2}} \frac{1-p}{p} u(\infty) .
$$

As already pointed out, the above equation has a unique solution $u(\infty)$ in $\left[w_{*}, u_{0}\right]$, namely $u(\infty)=u_{0}$ so $u(z) \rightarrow u_{0}$ as $z \rightarrow \infty$. Going back to the ODE for all $z \rightarrow \infty$ and not only along the subsequence, we actually obtain $z^{2} u^{\prime \prime}(z) \rightarrow 0$ as well.

Let now $1 \leq z_{1}<z_{2}$. Using the global uniqueness argument above, it is easy to see that $u\left(z_{1}\right)<u_{0}$. If $u\left(z_{1}\right) \geq u\left(z_{2}\right)$, since $\lim u_{z \rightarrow \infty} u(z)=u_{0}$, we conclude that there exists $z_{3}$ large enough such that $u\left(z_{2}\right) \leq u\left(z_{1}\right)<u\left(z_{3}\right)$. This means that $u$ would necessarily have an interior minimum in $\left[z_{1}, z_{3}\right]$, which is impossible. Therefore, $u$ is strictly increasing. $\diamond$ 
Proof of Proposition 4.1: The limits of $\hat{c}$ and $\hat{\pi}$ as $z \rightarrow \infty$ are easy consequences of their representations (20) and (19) together with the fact that $z u^{\prime}(z), z^{2} u^{\prime \prime}(z) \rightarrow 0$ as $z \rightarrow \infty$. Taking derivatives of the (HJB) in the form

$$
\frac{d}{d z}\left(-p(1-p) u+2 p z u^{\prime}+z^{2} u^{\prime \prime}\right)=\frac{d}{d z}\left(\frac{1}{2} \frac{\alpha^{2}}{\sigma^{2}} \frac{\left((1-p) u-z u^{\prime}\right)^{2}}{-\beta u+\tilde{V}((1-p) u-(z-1) u))}\right),
$$

and using again that $z u^{\prime}(z), z^{2} u^{\prime \prime}(z) \rightarrow 0$ as $z \rightarrow \infty$, we conclude that we also have

$$
z^{3} u^{\prime \prime \prime}(z) \rightarrow 0, \quad z \rightarrow \infty .
$$

We can now use the representations (19) and (20) and take their derivatives to obtain (30). The qualitative description of consumption (31) is obtained from the representation (20) using the fact that $u^{\prime} \geq 0$ and $u \leq u_{0} . \diamond$

\subsection{Optimal policies and verification}

Proposition 5.2 Let $\theta(x, n)$ and $\gamma(x, n)$ two Lipschitz functions in both arguments on the twodimensional domain $\left\{(x, n) \in \mathbb{R}^{2} ; \quad x \leq n\right\}$. The closed loop state equation (7) corresponding to $\theta_{s}=\theta\left(X_{s}, N_{s}\right)$ and $\gamma_{s}=\gamma\left(X_{s}, N_{s}\right)$, which means the $S D E$

$$
\left\{\begin{array}{l}
X_{t}=x+\int_{0}^{t} \theta\left(X_{s}, N_{s}\right)\left(\alpha d s+\sigma d W_{s}\right)-\int_{0}^{t} \gamma\left(X_{s}, N_{s}\right) d s-\lambda\left(N_{t}+\int_{0}^{t} \gamma\left(X_{s}, N_{s}\right) d s-n\right) \\
N_{t}=\max _{0 \leq s \leq t}\left\{\left(X_{s}+\int_{0}^{s} \gamma\left(X_{u}, N_{u}\right) d u\right) \vee n\right\}-\int_{0}^{t} \gamma\left(X_{s}, N_{s}\right) d s
\end{array}\right.
$$

has a unique strong solution $(X, N)$.

Proof: Consider the operator

$$
(Y, L) \rightarrow(X, N)
$$

defined by

$$
\left\{\begin{array}{l}
X_{t}=x+\int_{0}^{t} \theta\left(Y_{s}, L_{s}\right)\left(\alpha d s+\sigma d W_{s}\right)-\int_{0}^{t} \gamma\left(Y_{s}, L_{s}\right) d s-\lambda\left(N_{t}+\int_{0}^{t} \gamma\left(Y_{s}, L_{s}\right) d s-n\right), \\
N_{t}=\max _{0 \leq s \leq t}\left\{\left(X_{s}+\int_{0}^{s} \gamma\left(Y_{u}, L_{u}\right) d u\right) \vee n\right\}-\int_{0}^{t} \gamma\left(Y_{s}, L_{s}\right) d s
\end{array}\right.
$$

In other words, we obtain $(X, N)$ from $(Y, L)$ by solving the state equation $(7)$ for $\theta_{s}=\theta\left(Y_{s}, L_{s}\right)$ and $\gamma_{s}=\gamma\left(Y_{s}, L_{s}\right)$. According to Proposition 2.2, the solution $(X, N)$ is given by

$$
\begin{aligned}
X_{t}=x & +\int_{0}^{t} \theta\left(Y_{s}, L_{s}\right)\left((\alpha-\nu) d s+\sigma d W_{s}\right)-\int_{0}^{t} \gamma\left(Y_{s}, L_{s}\right) d s- \\
& -\frac{\lambda}{1+\lambda} \max _{0 \leq s \leq t}\left[\int_{0}^{s} \theta\left(Y_{u}, L_{u}\right)\left((\alpha-\nu) d u+\sigma d W_{u}\right)-i\right]^{+},
\end{aligned}
$$

and

$$
N_{t}=n+\frac{1}{1+\lambda} \max _{0 \leq s \leq t}\left[\int_{0}^{s} \theta\left(Y_{u}, L_{u}\right)\left((\alpha-\nu) d u+\sigma d W_{u}\right)-i\right]^{+}-\int_{0}^{t} \gamma\left(Y_{s}, L_{s}\right) d s .
$$

Now we can use the usual estimates in the Itô theory of stochastic differential equations to obtain

$$
\mathbb{E}\left[\max _{0 \leq s \leq t}\left\|\left(X_{s}^{1}-X_{s}^{2}, N_{s}^{1}-N_{s}^{2}\right)\right\|^{2}\right] \leq C^{*}(L, T) \int_{0}^{t} \mathbb{E}\left[\left\|\left(Y_{s}^{1}-Y_{s}^{2}, L_{s}^{1}-L_{s}^{2}\right)\right\|^{2}\right] d s, \quad 0 \leq t \leq T
$$

for each fixed $T>0$, where $C^{*}(L, T)<\infty$ is a constant depending on the Lipschitz constant of $\theta$ and $\gamma$ as well as the time horizon $T$. This allows to prove path-wise uniqueness using Gronwall's Inequality, and also to prove existence using a Picard Iteration. $\diamond$ 
Proof of Proposition 3.1: From Proposition 4.1 we can easily see that

$$
\hat{\theta}(x, n) \triangleq\left\{\begin{array}{l}
x \hat{\pi}(n / x), \quad 0<x \leq n \\
0, \quad x \leq 0, \quad x \leq n
\end{array}\right.
$$

and

$$
\hat{\gamma}(x, n) \triangleq\left\{\begin{array}{l}
x \hat{c}(n / x), \quad 0<x \leq n \\
0, \quad x \leq 0, \quad x \leq n
\end{array}\right.
$$

are globally Lipschitz in the domain $x \leq n<\infty$. Therefore, according to Proposition (5.2) the equation has a unique solution $\hat{X} \leq \hat{N}$. It only remains to prove that $0<\hat{X}$ in order to finish the proof of Proposition 3.1, and this is done in the Proposition below. $\diamond$

Proposition 5.3 Let $0<x \leq n$. Assume that the predictable process $(\pi, c)$ satisfies the integrability condition

$$
\mathbb{P}\left(\int_{0}^{t}\left(\left|\pi_{u}\right|^{2}+c_{u}\right) d u<\infty, \quad(\forall) \quad 0 \leq t<\infty\right)=1 .
$$

If $(X, N)$ is a solution to the equation

$$
\left\{\begin{array}{l}
d X_{t}=X_{t}\left(\pi_{t} \alpha-c_{t}\right) d t+\pi_{t} X_{t} \sigma d W_{t}-\lambda\left(d N_{t}+c_{t} X_{t} d t\right), \quad X_{0}=x \\
N_{t}=\sup _{0 \leq s \leq t}\left\{\left(X_{s}+\int_{0}^{s} c_{u} X_{u} d u\right) \vee n\right\}-\int_{0}^{t} c_{s} X_{s} d s
\end{array}\right.
$$

then

$$
N_{t} \geq X_{t}>0, \quad 0 \leq t<\infty
$$

Proof: Denote by $\tau \triangleq \min \left\{t \geq 0: X_{t}=0\right\}$. We can apply Itô's formula to $Y_{t}=\log \left(X_{t}\right)$ and take into account that $X=N$ on the support of $d M$ to obtain

$Y_{t}=\log x+R_{t}-\lambda \int_{0}^{t} \frac{d M_{s}}{N_{s}}=\log x+R_{t}-\lambda \int_{0}^{t} c_{s} \frac{X_{s}}{N_{s}} d s-\lambda\left(\log N_{t}-\log m\right) \geq \log x+L_{t}-\lambda \sup _{0 \leq s \leq t}\left[Y_{t}-\log m\right]^{+}$,

where

$$
R_{t} \triangleq \int_{0}^{t}\left(\pi_{s} \alpha-c_{s}-\frac{1}{2} \pi_{s}^{2} \sigma^{2}\right) d s+\int_{0}^{t} \pi_{s} \sigma d W_{s}, \quad 0 \leq t
$$

and

$$
L_{t} \triangleq R_{t}-\lambda \int_{0}^{t} c_{s} \frac{X_{s}}{N_{s}} d s, 0 \leq t<\tau
$$

Observing that, because $0 \leq X_{s} / N_{s} \leq 1$, we have

$$
\lim _{t \nearrow \tau} L_{t}>-\infty \text { on }\{\tau<\infty\}
$$

we can then obtain that $\tau=\infty$. $\diamond$

Proof of Theorem 3.2: According to Lemma 3.1, the process

$$
V_{t}=\int_{0}^{t} e^{-\beta s} U\left(\gamma_{s}\right) d s+e^{-\beta t} v\left(X_{t}, N_{t}\right), t \geq 0,
$$

is a local supermartingale for each admissible control and a local martingale for the feedback control $(\hat{\theta}, \hat{\gamma})$.

1. If $p>1$, then, for a sequence of stopping times $\tau_{k}$ we have

$$
v(x, n)=\mathbb{E}\left[\int_{0}^{\tau_{k}} e^{-\beta s} U\left(\hat{\gamma}_{s}\right) d s+e^{-\beta \tau_{k}} v\left(\hat{X}_{\tau_{k}}, \hat{N}_{\tau_{k}}\right)\right] \leq \mathbb{E}\left[\int_{0}^{\tau_{k}} e^{-\beta s} U\left(\hat{\gamma}_{s}\right) d s\right] .
$$

Letting $k \rightarrow \infty$ and using Monotone Convergence, we get 


$$
v(x, n) \leq \mathbb{E}\left[\int_{0}^{\infty} e^{-\beta s} U\left(\hat{\gamma}_{s}\right) d s\right] .
$$

Now, let $(\theta, \gamma) \in \mathcal{A}(x, n)$ be admissible controls . It is easy to see from Proposition 2.2 that $(\theta, \gamma) \in \mathcal{A}(x+\varepsilon, n+\varepsilon)$, and the wealth $X$ corresponding to $(\theta, \gamma)$ starting at $x+\varepsilon$ with highwatermark $i$ actually satisfies $X>\varepsilon$. Using the local supermartingale property along the solution $(X, N)$ starting at $(x+\varepsilon, n+\varepsilon)$ with controls $(\theta, \gamma)$, we obtain

$$
v(x+\varepsilon, n+\varepsilon) \geq \mathbb{E}\left[\int_{0}^{\tau_{k}} e^{-\beta s} U\left(\gamma_{s}\right) d s+e^{-\beta \tau_{k}} v\left(X_{\tau_{k}}, N_{\tau_{k}}\right)\right] .
$$

However, since $X>\varepsilon$ then we obtain

$$
|v(X, N)| \leq C \varepsilon^{1-p}
$$

where $C$ is a bound on $|u|$. Therefore, we can let again $k \rightarrow \infty$ and use Monotone convergence together with Bounded Convergence theorem (respectively for the two terms on the right hand side) to obtain

$$
v(x+\varepsilon, n+\varepsilon) \geq \mathbb{E}\left[\int_{0}^{\infty} e^{-\beta s} U\left(\gamma_{s}\right) d s\right],
$$

for all $(\theta, c) \in \mathcal{A}(x, n)$. This means that

$$
v(x+\varepsilon, n+\varepsilon) \geq \sup _{(\theta, \gamma) \in \mathcal{A}(x, n)} \mathbb{E}\left[\int_{0}^{\infty} e^{-\beta s} U\left(\gamma_{s}\right) d s\right]=V(x, n)
$$

and the conclusion follows from letting $\varepsilon \searrow 0$.

2. Let $p<1$. Then by using the local supermartingale property we obtain

$$
v(x, n) \geq \mathbb{E}\left[\int_{0}^{\tau_{k}} e^{-\beta s} U\left(\gamma_{s}\right) d s+e^{-\beta \tau_{k}} v\left(X_{\tau_{k}}, N_{\tau_{k}}\right)\right] \geq \mathbb{E}\left[\int_{0}^{\tau_{k}} e^{-\beta s} U\left(\gamma_{s}\right) d s\right] .
$$

Letting $k \rightarrow \infty$ we get

$$
v(x, n) \geq \mathbb{E}\left[\int_{0}^{\infty} e^{-\beta s} U\left(\gamma_{s}\right) d s\right]
$$

for each $(\theta, \gamma) \in \mathcal{A}(x, n)$.

Now, for the optimal $(\hat{\pi}, \hat{c})$ (in proportion form) we have

$$
v(x, n)=\mathbb{E}\left[\int_{0}^{\tau_{k}} e^{-\beta s} U\left(\hat{c}_{s} \hat{X}_{s}\right) d s+e^{-\beta \tau_{k}} v\left(\hat{X}_{\tau_{k}}, \hat{N}_{\tau_{k}}\right)\right] .
$$

If we can show that

$$
\mathbb{E}\left[e^{-\beta \tau_{k}} v\left(\hat{X}_{\tau_{k}}, \hat{N}_{\tau_{k}}\right)\right] \rightarrow 0
$$

then we use Monotone Convergence to obtain

$$
v(x, n)=\mathbb{E}\left[\int_{0}^{\infty} e^{-\beta s} U\left(\hat{c}_{s} \hat{X}_{s}\right) d s\right]
$$

and finish the proof. Let us now prove (55).

The value function $v_{0}(x, n) \triangleq u_{0} x^{1-p}$ corresponding to $\lambda=0$ is a supersolution of the HJB since the constant function $u_{0}$ is a supersolution to equation (35). Using Lemma 3.1 for the function $v_{0}$ and denoting by

$$
Z_{t} \triangleq e^{-\beta t} v_{0}\left(\hat{X}_{t}, \hat{N}_{t}\right)=u_{0} e^{-\beta t}\left(\hat{X}_{t}\right)^{1-p}
$$


we obtain that

$$
d Z_{t}+e^{-\beta t} \frac{\left(\hat{c} \hat{X}_{t}\right)^{1-p}}{1-p} d t=d Z_{t}+\frac{\hat{c}^{1-p}}{(1-p) u_{0}} Z_{t} d t \leq(1-p) \hat{\pi} \sigma Z_{t} d W_{t}
$$

Recall that, from Proposition 4.1, we have that $\hat{c} \geq c_{0}$. This means that, if we denote by

$$
\delta \triangleq \frac{c_{0}^{1-p}}{(1-p) u_{0}}>0
$$

then we have

$$
d Z_{t}+\delta Z_{t} d t \leq(1-p) \hat{\pi} \sigma Z_{t} d W_{t}
$$

Denoting by

$$
L_{t} \triangleq \int_{0}^{t}(1-p) \hat{\pi} \sigma d W_{u}
$$

we can easily integrate the formal inequality above to obtain

$$
Z_{t} \leq Z_{0} e^{L_{t}-\frac{1}{2}\langle L\rangle_{t}-\delta t}
$$

Since $\hat{\pi}$ is actually bounded, we conclude that there exists a constant $C<\infty$ such that $\langle L\rangle_{t} \leq C t$, so actually we have that

$$
Z_{t} \leq Z_{0} e^{L_{t}-\left(\frac{1}{2}+\frac{\delta}{C}\right)\langle L\rangle t} .
$$

Using the exponential distribution of maximum of the Brownian Motion with drift together with the DDS representation, we obtain that

$$
\mathbb{E}\left[\sup _{0 \leq t<\infty} Z_{t}\right]<\infty
$$

Taking into account that

$$
e^{-\beta t} v\left(\hat{X}_{t}, \hat{N}_{t}\right) \leq Z_{t} \rightarrow 0 \text { a.s for } t \rightarrow \infty,
$$

we obtain (55) and the proof is complete.

\section{References}

[1] V. Agarwal, N. Daniel and N. Naik Role of Managerial Incentives and Discretion in Hedge Fund Performance Journal of Finance, forthcoming.

[2] J. Aragon and J. Qian, The Role of High-Watermarks in Hedge-Fund Compensation preprint, 2007

[3] Crandall, M., H. Ishii, \& P.-L. Lions, User's guide to viscosity solutions of second-order partial differential equations, Bull. Amer. Math. Soc., Vol. 27 (1992), 1-67

[4] J. Cvitanic, and I. Karatzas On Portfolio Optimization under Drawdown Constraints, IMA Lecture Notes in Mathematics and Applications, Vol. 65 (1995), 77-88.

[5] M.H.A. Davis and A. Norman, Portfolio selection with transaction costs, Math. Oper. Res., Vol $15,(1990) 676-713$

[6] R. Elie and N. Touzi, Optimal Lifetime Consumption and Investment under a Draw-Down Constraint, Finance and Stochastics, Vol. 12 (2008), 299-330. 
[7] WN Goetzmann, JE Ingersoll Jr, SA Ross High-water marks and hedge fund management contracts The Journal of Finance, 2003

[8] S. Grossman and Z. Zhou Optimal Investment Strategies for Controlling Drawdawns, Mathematical Finance, Vol. 3 (1993), 241-276.

[9] P. Guasoni and J. Obloj, The incentives of hedge fund fees and high-water-marks, preprint, 2009.

[10] K. Janecek and S. Shreve, Asymptotic analysis for optimal investment and consumption with transaction costs, Finance and Stochastics, Vol 8 (2004), 181-206

[11] K. Janeček and S. Shreve, Futures Trading with Transaction Costs, Illinois Journal of Mathematics, to appear (2011)

[12] R. C. Merton, Lifetime portfolio selection under uncertainty: the continuous-time case Rev. Econom. Statist., (1969), 247-257

[13] R. C. Merton, Optimum consumption and portfolio rules in a continuous-time model, J. Economic Theory, (1971), 373-413

[14] S, Panagea and M, M. Westerfield, High-Water Marks: High Risk Appetites? Convex Compensations, Long Horizons and Portfolio Choice The Journal of Finance", Vol. 64, No. 1, pp. 1-36.

[15] H. Roche, Optimal Consumption and Investment Strategies under Wealth Racheting, preprint 2006 .

[16] S. Shreve and M. Soner, Optimal investment and consumption with transaction costs, Ann. Appl. Probab., Vol 4, (1994), 609-692

[17] Whalley, A.E. and Wilmott, P. , An asymptotic analysis of an optimal hedging model for option pricing under transaction costs, Mathematical Finance, vol 7(1997), 307-324 